\title{
Myocardial Galectin-3 Expression Is Associated with Remodeling of the Pressure-Overloaded Heart and May Delay the Hypertrophic Response without Affecting Survival, Dysfunction, and Cardiac Fibrosis
}

\author{
Olga Frunza, Ilaria Russo, Amit Saxena, Arti V. Shinde, Claudio Humeres, Waqas Hanif, Vikrant Rai, Ya Su, and
}

Nikolaos G. Frangogiannis

From the Department of Medicine (Cardiology), Wilf Family Cardiovascular Research Institute, Albert Einstein College of Medicine, Bronx, New York

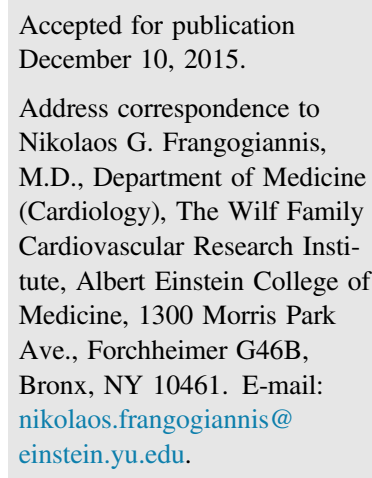

Address correspondence to Nikolaos G. Frangogiannis, M.D., Department of Medicine (Cardiology), The Wilf Family Cardiovascular Research Institute, Albert Einstein College of Medicine, 1300 Morris Park Ave., Forchheimer G46B, Bronx, NY 10461. E-mail: nikolaos.frangogiannis@ einstein.yu.edu.

\begin{abstract}
The $\beta$-galactoside-binding animal lectin galectin- 3 is predominantly expressed by activated macrophages and is a promising biomarker for patients with heart failure. Galectin-3 regulates inflammatory and fibrotic responses; however, its role in cardiac remodeling remains unclear. We hypothesized that galectin-3 may be up-regulated in the pressure-overloaded myocardium and regulate hypertrophy and fibrosis. In normal mouse myocardium, galectin-3 was constitutively expressed in macrophages and was localized in atrial but not ventricular cardiomyocytes. In a mouse model of transverse aortic constriction, galectin-3 expression was markedly up-regulated in the pressure-overloaded myocardium. Early up-regulation of galectin- 3 was localized in subpopulations of macrophages and myofibroblasts; however, after 7 to 28 days of transverse aortic constriction, a subset of cardiomyocytes in fibrotic areas contained large amounts of galectin-3. In vitro, cytokine stimulation suppressed galectin-3 synthesis by macrophages and cardiac fibroblasts. Correlation studies revealed that cardiomyocytebut not macrophage-specific galectin-3 localization was associated with adverse remodeling and dysfunction. Galectin-3 knockout mice exhibited accelerated cardiac hypertrophy after 7 days of pressure overload, whereas female galectin-3 knockouts had delayed dilation after 28 days of transverse aortic constriction. However, galectin-3 loss did not affect survival, systolic and diastolic dysfunction, cardiac fibrosis, and cardiomyocyte hypertrophy in the pressure-overloaded heart. Despite its potential role as a prognostic biomarker, galectin-3 is not a critical modulator of cardiac fibrosis but may delay the hypertrophic response. (Am J Pathol 2016, 186: 1114-1127; http://dx.doi.org/ 10.1016/j.ajpath.2015.12.017)
\end{abstract}

Galectin-3, a member of the galectin family, is a $30-\mathrm{kDa}$ $\beta$-galactoside-binding lectin with a broad repertoire of cellular functions. Although often viewed as a specific marker for macrophages, ${ }^{1,2}$ galectin- 3 can also be expressed by other cell types on activation, including epithelial cells, ${ }^{3}$ fibroblasts, ${ }^{4}$ eosinophils, ${ }^{5}$ and endothelial cells. ${ }^{6}$ Galectin-3 exerts a wide range of actions, modulating proliferation, survival, differentiation, and gene expression in many different cell types. The promiscuous subcellular localization of galectin-3 may account for its functional flexibility and explain its diverse and context-dependent in vivo actions. Galectin-3 can be localized in the cytoplasm, cell surface, or the nucleus, or it can be secreted and incorporated into the extracellular matrix ${ }^{7}$ that modulates cell behavior both through intracellular effects on signaling cascades and through matricellular actions.

Extensive in vitro and in vivo evidence implicates galectin-3 in tissue inflammation and fibrosis. Intracellular galectin-3 plays a critical role in regulating phagocytotic

Supported by NIH grants R01 HL76246, R01 HL85440, and T32HL007675 (O.F.) and an American Heart Association Founders affiliate postdoctoral award (A.V.S.).

Disclosures: None declared. 
activity in macrophages and drives alternative macrophage activation. ${ }^{8}$ In vivo studies have produced conflicting results, suggesting that galectin-3 expression may mediate both proinflammatory ${ }^{9}$ and anti-inflammatory actions. ${ }^{10}$ Profibrotic effects of galectin-3 have been documented in experimental models of hepatic, renal, and vascular fibrosis $^{11-13}$ and may be mediated through galectin3 -induced acquisition of a fibrogenic macrophage phenotype $^{8}$ or by direct actions of secreted galectin-3 on fibroblasts. ${ }^{14}$

A growing body of evidence suggests a role for members of the galectin family in cardiovascular disease. ${ }^{15,16}$ Over the past decade, clinical studies have documented marked elevations of circulating galectin-3 concentrations in patients with heart failure ${ }^{17}$ and have generated significant interest in the role of galectin-3 as a biomarker. In patients with acute or chronic heart failure, plasma galectin- 3 concentrations were shown to predict mortality and adverse outcome in a variety of clinical settings. ${ }^{17-20}$ Despite the growing interest in the role of galectin-3 as a circulating biomarker, little is known about the mechanisms of galectin3 regulation in the failing heart and its potential role in the pathogenesis of heart failure. ${ }^{16}$ In a transgenic rat model of cardiac hypertrophy, galectin-3 expression was predominantly localized in activated cardiac macrophages and identified animals prone to develop heart failure. ${ }^{21}$ Moreover, studies in rodent models of cardiac remodeling showed that galectin-3 loss reduces fibrosis, attenuating myocardial dysfunction. ${ }^{22,23}$ On the basis of these studies, it has been suggested that galectin-3 may be a promising therapeutic target in patients with heart failure. However, both investigations were limited to male animals.

Considering the emerging interest in the role of galectin-3 in heart failure, we designed a systematic investigation of the effects of galectin-3 on cardiac remodeling, using both male and female mice subjected to standardized pressure overload protocols. Our findings suggest that galectin-3 is markedly upregulated in the pressure-overloaded myocardium, and its expression is associated with adverse cardiac remodeling. However, despite its potential role as a biomarker, galectin-3 did not affect survival, systolic and diastolic dysfunction, and fibrosis in the pressure-overloaded heart. Genetic loss of galectin-3 had relatively subtle and transient effects on the remodeling myocardium, accelerating hypertrophy and delaying dilation in female animals.

\section{Materials and Methods}

\section{Animal Protocols}

Animal experiments were conducted in accordance with the NIH Guide for the Care and Use of Laboratory Animals ${ }^{24}$ and were approved by the Albert Einstein College of Medicine Institutional Review Boards. Male and female, 2- to 4-monthold wild-type (WT; $n=50$ ) and galectin-3 knockout (KO; $n=46$ ) mice in a C57/BL6 background were purchased from
The Jackson Laboratory (Bar Harbor, ME). Mice were anesthetized with inhaled isoflurane. Aortic banding was achieved by creating a constriction between the right innominate and left carotid arteries as previously described. ${ }^{25,26}$ The degree of pressure overload was assessed by measuring right-to-left carotid artery flow velocity ratio after constricting the transverse aorta. At the end of the experiment, the heart was excised, fixed in zinc-formalin, and embedded in paraffin for histologic studies. Hearts used for histologic analysis were sectioned from base to apex at $250-\mu \mathrm{m}$ intervals. Mice used for histology underwent 28 days of banding $(n=10$ mice per group). As a control, a sham operation without aortic constriction was performed on age-matched mice $(n=4$ per group). To study galectin-3 localization in normal mouse tissues, four additional WT mice (4 months of age) were sacrificed. The spleen, liver, kidney, bowel, lung, and heart were harvested, fixed in zinc-formalin, and embedded in paraffin. Additional groups of male and female WT and galectin-3 KO mice underwent transverse aortic constriction (TAC) protocols for 56 days (WT, $n=20 ; \mathrm{KO}, n=16$ ).

\section{Echocardiography}

Short-axis M-mode echocardiography was performed before instrumentation and after 7 or 28 days of TAC with the use of the Vevo 770 system (VisualSonics, Toronto, ON, Canada) as previously described. ${ }^{25-27}$ The following variables were measured as indicators of function and remodeling: left ventricular (LF) end-diastolic diameter, LF end-systolic diameter, LV end-diastolic volume (LVEDV), LV end-systolic volume, ejection fraction, and LV mass. Mice enrolled in the 56-day TAC study had echocardiography at baseline and after 7, 28, and 56 days of TAC. The percentage of change for these variables was calculated for every time point studied with the use of the following formula:

$\Delta$ LVEDV7d $=($ LVEDV7d - LVEDVpre $) \times 100 /$ LVEDVpre $(1)$

\section{Doppler Echocardiography and Tissue Doppler Imaging}

Mice undergoing 56-day TAC protocols had Doppler echocardiography and tissue Doppler imaging at baseline and after 7, 28, and 56 days of TAC with the use of the Vevo 2100 system (VisualSonics). Transmitral LV inflow velocities were measured from apical four-chamber view by pulsed-wave Doppler. Peak early E (E-wave) and late A (A-wave) filling velocities and E/A ratio were measured according to the guidelines of the American Society of Echocardiography. ${ }^{28}$ Tissue Doppler imaging of the mitral annulus was obtained from the apical four-chamber view. A 1.0-mm sample volume was placed sequentially at the medial mitral annulus. Analysis was performed for systolic velocity and for the early $\left(\mathrm{E}^{\prime}\right)$ and late diastolic velocity $\left(\mathrm{A}^{\prime}\right)$. The mitral inflow $\mathrm{E}$ velocity to tissue Doppler $E^{\prime}$ wave velocity ratio $\left(\mathrm{E}^{\prime} \mathrm{E}^{\prime}\right)$ and tissue Doppler early $E^{\prime}$ velocity to tissue Doppler late $A^{\prime}$ velocity ratio were 
calculated. All Doppler spectra were recorded for three to five cardiac cycles at a sweep speed of $100 \mathrm{~mm} / \mathrm{second}$. The color Doppler preset was at a Nyquist limit of $0.44 \mathrm{~m} / \mathrm{second}$. The off-line analysis was performed by a sonographer (I.R.) blinded to study groups.

\section{Immunohistochemistry, Dual Immunofluorescence, and Quantitative Histology}

To study galectin-3 localization, histologic sections were stained immunohistochemically with a rat anti-mouse galectin-3 antibody (Mac2; dilution 1:200; Cedarlane Laboratories, Burlington, ON, Canada) as previously described. ${ }^{26}$ Staining was performed with the use of a peroxidase-based technique with the Vectastain ELITE rat kit (Vector Laboratories Inc., Burlingame, CA). Sections were pretreated with a solution of $3 \%$ hydrogen peroxide to inhibit endogenous peroxidase activity and incubated with $2 \%$ rabbit serum (Vectastain ELITE kit; Vector Laboratories Inc.) to block nonspecific protein binding. Peroxidase activity was detected with diaminobenzidine with nickel. Negative controls were performed with omission of the primary antibody. Tissues from galectin-3 KO mice were used as additional negative controls for Mac2 immunohistochemistry. Slides were counterstained with eosin. Immunofluorescence staining was performed with the use of the following antibodies: rat anti-mouse Mac-2 antibody, anti- $\alpha$-smooth muscle actin ( $\alpha$-SMA) antibody (dilution 1:200; Sigma-Aldrich, St. Louis, MO), rabbit antiCD68 antibody (dilution 1:200; Abcam, Cambridge, MA), and rabbit anti-pancytokeratin antibody (dilution 1:250; Bioss, Woburn, MA). Cardiomyocytes were outlined with wheat germ agglutinin (WGA) histochemistry, as previously described with an Alexa Fluor 594 Conjugate (dilution 1:500; Life Technologies, Carlsbad, CA). ${ }^{27}$ For studies that examined the cellular localization of galectin-3, dual immunofluorescence that combined Mac-2/a-SMA, Mac-2/CD-68, and Mac-2/WGA staining was performed. Myofibroblasts were identified as spindle-shaped $\alpha$-SMA ${ }^{+}$cells located outside the vascular media. Quantitative assessment of the density of galectin-3-expressing myofibroblasts, macrophages, and cardiomyocytes was performed by counting the number of cells that exhibited dual fluorescence in five random fields from two different sections from each heart with the use of ImagePro Plus software version 4.5.0.29 (Media Cybernetics, Rockville, MD). Cell density was expressed as cells $/ \mathrm{mm}^{2}$. Quantitative histology was performed by an investigator (O.F.) blinded to the genotype of the animal, to the time point studied, and to the corresponding echocardiographic data.

\section{Collagen Staining and Assessment of Cardiomyocyte Size}

Collagen staining was performed with Sirius Red as previously described. ${ }^{29}$ The collagen-stained area was quantitatively assessed with Image Pro Plus software and expressed as a percentage of the total myocardial area. Assessment of cardiomyocyte size was performed by measuring the area of 25 random cardiomyocytes, cut in cross-section, from five different regions of slides stained for WGA staining. Cardiomyocyte area was expressed in $\mu \mathrm{m}^{2}$.

\section{Isolation and Stimulation of Mouse Cardiac Fibroblasts in Collagen Pads}

Expression of galectin-3 was studied in cardiac fibroblasts cultured in collagen pads as previously described. ${ }^{29-31}$ Collagen matrix was prepared by diluting a stock solution of rat $3 \mathrm{mg} / \mathrm{mL}$ collagen I (GIBCO Invitrogen Corporation, Carlsbad, CA) with $2 \times$ Dulbecco's modified Eagle's medium and distilled water for a final concentration of $1 \mathrm{mg} / \mathrm{mL}$ collagen. Cardiac fibroblast cell suspensions were mixed with collagen solution to achieve the final concentration of $3 \times 10^{5}$ cells $/ \mathrm{mL}$. Subsequently, $500 \mu \mathrm{L}$ of this suspension was divided into aliquots in a 24-well culture plate (BD Falcon, San Jose, CA) and allowed to polymerize at $37^{\circ} \mathrm{C}$ for 15 minutes. After polymerization, pads were released from wells, transferred to 6-well culture plate (BD Falcon), and cultured in $1 \times$ Dulbecco's modified Eagle's medium for 24 hours in the presence or absence of transforming growth factor (TGF)- $\beta 1$ or IL-1 $\beta$. After 24 hours the pads were fixed in formalin and embedded in paraffin for immunohistochemical staining. Dual staining for the fibroblast marker transcription factor 21 (Tcf21; dilution 1:100; using a rabbit anti-Tcf21 antibody from Abcam, Cambridge, UK) and galectin-3 was used to identify galectin-3-expressing fibroblasts. The number of galectin- $3^{+}$ fibroblasts was quantitatively assessed with Image Pro software.

\section{Sorting, Isolation, and Stimulation of Mouse Splenic Macrophages}

To examine the effects of cytokines on expression of galectin- 3 by macrophages, $\mathrm{CD}_{11 \mathrm{~b}^{+}}$macrophages were isolated from the mouse spleen with the use of immunomagnetic sorting as previously described. ${ }^{32}$ The cells were stimulated with IL- $1 \beta$ and TGF- $\beta 1$ (R\&D Systems, Minneapolis, MN) for 4 hours. At the end of the experiment, cells were harvested for RNA extraction. To examine whether the effects of TGF- $\beta 1$ were mediated through Smad3, galectin-3 expression was compared between macrophages harvested from WT and Smad3 KO mice (from our own colony). ${ }^{33,34}$

\section{RNA Extraction and $q P C R$}

Total RNA isolated from macrophages was reverse transcribed to cDNA with the use of iScript cDNA synthesis kit (Bio-Rad Laboratories, Hercules, CA) according to the manufacturer's guidelines. Quantitative real-time PCR (qPCR) was performed with the SsoFast EvaGreen supermix (Bio-Rad Laboratories) method on the iQ5 Real-Time PCR Detection System (Bio-Rad Laboratories) for 40 cycles 

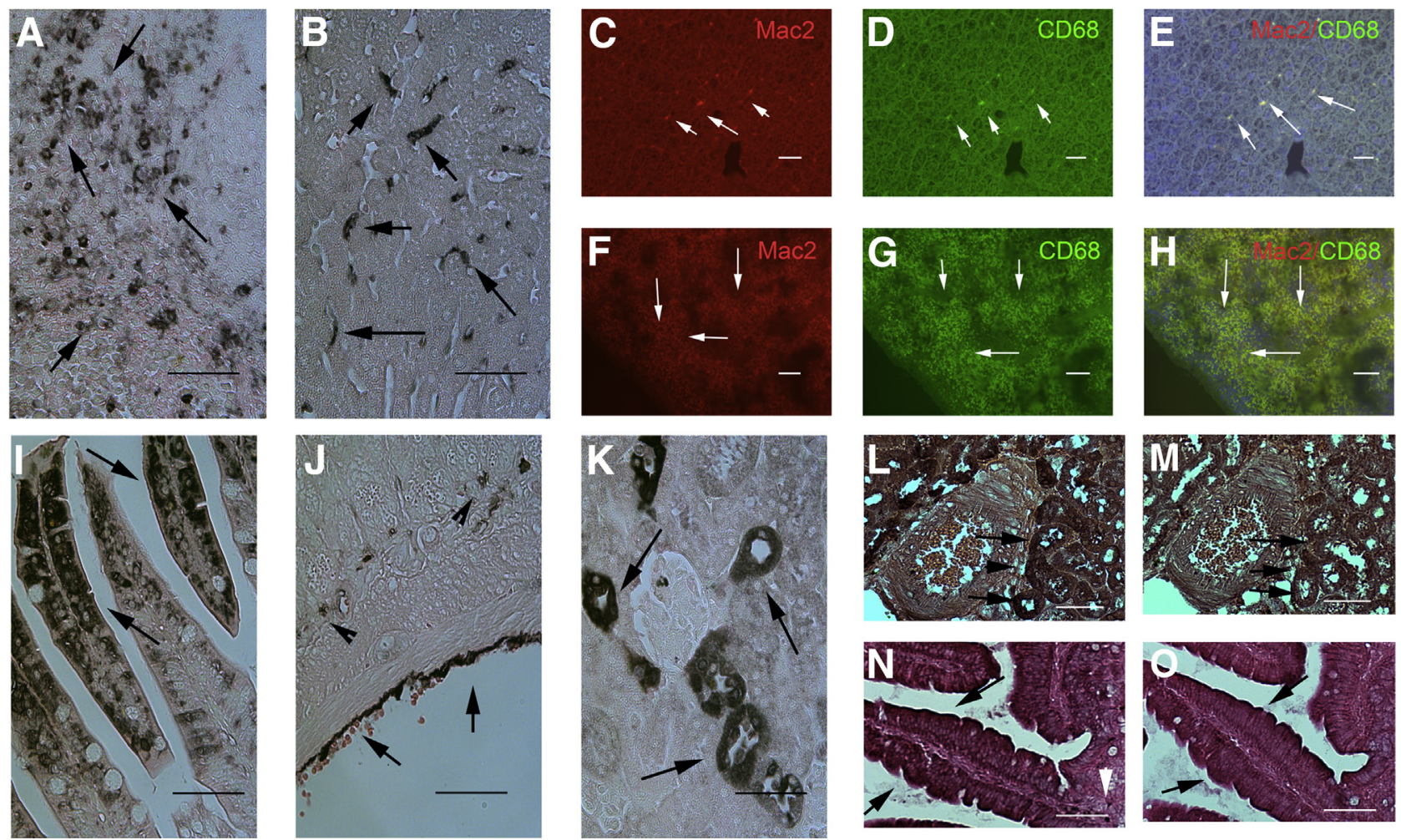

Figure 1 Galectin-3 immunohistochemistry in normal mouse tissues. A and B: In the mouse spleen (A) and liver (B), galectin-3 localizes in cells with morphologic characteristics of macrophages (arrows). C-H: Dual immunofluorescence for the anti-galectin-3 antibody Mac2 and CD68 localizes galectin-3 in liver $(\mathbf{C}-\mathbf{E})$ and spleen $(\mathbf{F}-\mathbf{H})$ macrophages (white arrows). I and J: However, in the bowel, galectin-3 staining also localizes in epithelial cells of the bowel lumen (I, arrows) and the serosa (J, arrows). Arrowheads show galectin- $3^{+}$cells with macrophage morphology in the bowel connective tissue. K: In the kidney, galectin-3 staining is noted in tubular epithelial cells (arrows). $\mathbf{L}-\mathbf{0}$ : Serial section staining of sections from the mouse kidney ( $\mathbf{L}$ and $\mathbf{M})$ and bowel (N and $\mathbf{0}$ ), localize galectin-3 immunoreactivity ( $\mathbf{L}$ and $\mathbf{N}$, arrows) in pancytokeratin ${ }^{+}$epithelial cells (M and $\mathbf{0}$, arrows). Counterstained with eosin. Scale bar $=60 \mu \mathrm{m}$.

at an annealing temperature $\left(\mathrm{T} \Delta \mathrm{Opt}{ }^{\circ} \mathrm{C}\right)$ specific for a primer pair. Primers were synthesized with Beacon designer from Premier Biosoft version 8.02 (Palo Alto, CA). Each sample was run in triplicate. The cycle threshold method with the use of $G A P D H$ as the reference gene was used for relative quantification of galectin-3 expression. The following sets of primers were used: galectin-3: forward, 5'-CCTTCTTGTAAGACATCCATT- ${ }^{\prime}$ and reverse, $5^{\prime}$-ACGAGCAGTCCTAAGATT-3'; GAPDH: forward, 5'-AACGACCCCTTCATTGACCT- $3^{\prime}$ and reverse, $5^{\prime}$-CACCAGTAGACTCCACGACA-3'.

\section{Statistical Analysis}

Data are expressed as means \pm SEM. For comparisons of two groups unpaired, two-tailed Student's $t$-test with the use of (when appropriate) Welch's correction for unequal variances was performed. The $U$ test was used for comparisons between two groups that did not show Gaussian distribution. For comparison of multiple groups one-way analysis of variance was performed, followed by $t$-test corrected for multiple comparisons (Student-Newman-Keuls). The Kruskall-Wallis test, followed by Dunn's multiple comparison posttest was used when one or more groups did not show Gaussian distribution. Mortality was compared with the log-rank test. Associations between echocardiographic and histologic end points were studied with Pearson's correlation analysis. Data are expressed as means \pm SEM. Statistical significance was set at 0.05 .

\section{Results}

\section{Galectin-3 Expression in Normal Mouse Tissues}

We used immunohistochemistry to study galectin-3 localization in normal mouse tissues. The specificity of the Mac2 antibody for galectin-3 was validated in experiments with the use of lung tissue from WT and galectin-3 KO mice (Supplemental Figure S1, A-C). Although often considered a relatively specific marker of macrophages, galectin-3 is expressed by nonmacrophage populations in several organs. In the spleen (Figure 1A) and liver (Figure 1B), galectin-3 was predominantly expressed by cells with macrophage morphology. Dual immunofluorescence for Mac2 and CD68 confirmed the expression of galectin-3 by hepatic (Figure 1, C-E) and spleen (Figure 1, F-H) macrophages. However, in the bowel, intestinal epithelial cells and serosal cells were strongly 

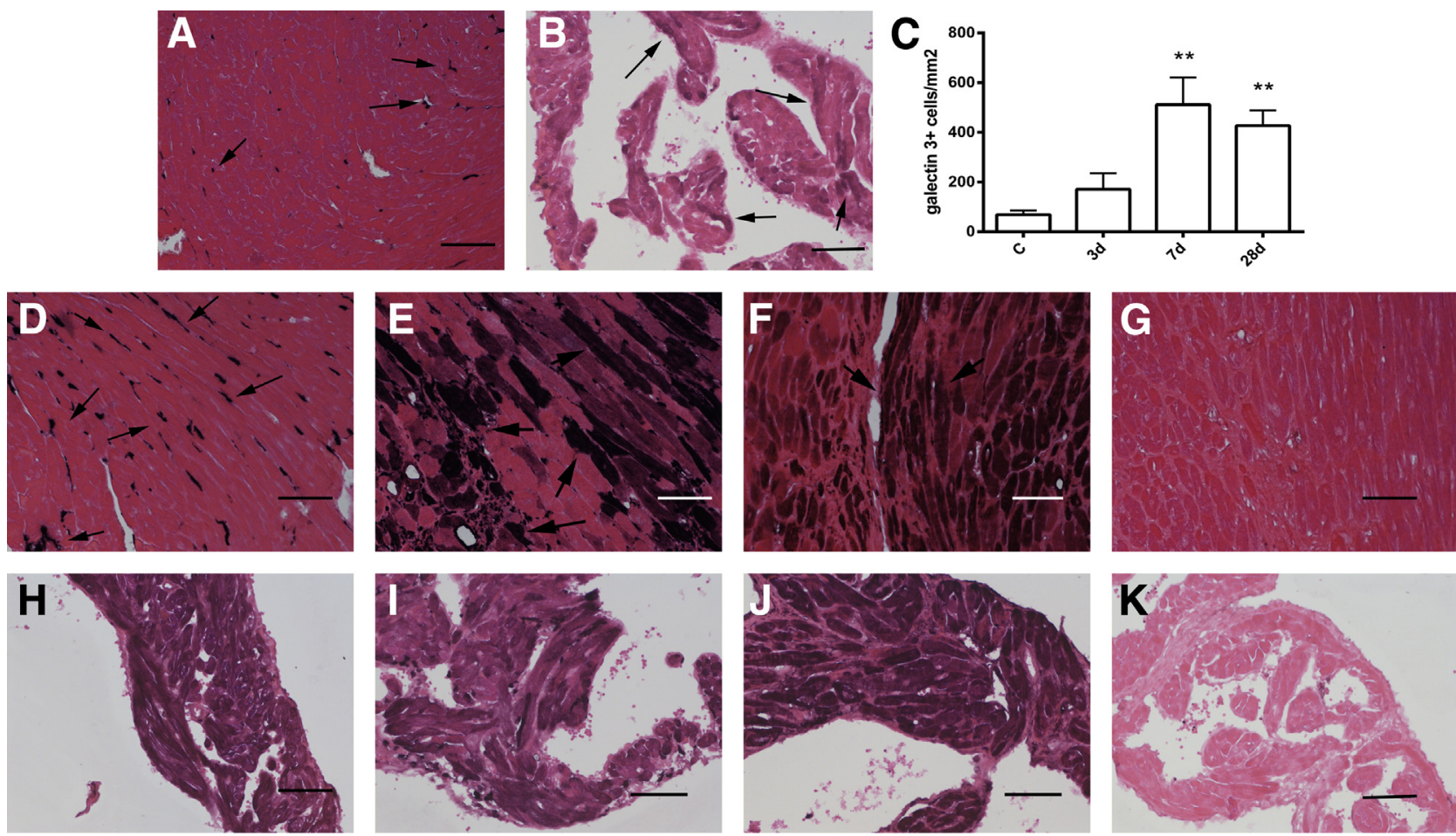

Figure 2 Galectin-3 up-regulation in the pressure-overloaded myocardium. A: In normal adult mouse left ventricle, galectin-3 localizes in interstitial cells with morphologic characteristics of macrophages (arrows). Ventricular cardiomyocytes do not express galectin-3. B: In control mouse atria, cardiomyocytes exhibit low-level galectin-3 immunoreactivity (arrows). C: The density of galectin- $3^{+}$cells in the left ventricular myocardium was markedly increased after pressure overload after 7 to 28 days of TAC. D: After 3 days of TAC, galectin-3 staining predominantly localizes in interstitial cells with morphologic characteristics of macrophages (arrows). E and F: After 7 to 28 days of TAC, intense galectin-3 staining also localizes in a subset of cardiomyocytes adjacent to areas of fibrosis (arrows). G: A section from a galectin-3 null animal after 28 days of TAC serves as a negative control, showing no galectin-3 staining, thus indicating the specificity of Mac2 immunohistochemistry for galectin-3. H-J: Atrial cardiomyocytes exhibit increased galectin-3 staining after 3 days (H), 7 days (I), and 28 days (J) of TAC. K: Galectin-3 null mice show no galectin-3 immunoreactivity after 28 days of TAC, confirming the specificity of immunohistochemical staining. Counterstained with eosin. Data are expressed as means \pm SEM. $n=4$ to 10 per group. ${ }^{* *} P<0.01$ versus control. Scale bar $=60 \mu \mathrm{m}$. C, control; TAC, transverse aortic constriction.

positive for galectin-3 (Figure 1, I and J). In the kidney, epithelial cells lining the distal convoluted tubules and collecting ducts in the cortex and the thick loops of Henle expressed large amounts of galectin-3 (Figure 1K). Serial section staining for pancytokeratin and Mac-2 localized galectin-3 in epithelial cells in the mouse kidney (Figure 1, $\mathrm{L}$ and $\mathrm{M}$ ) and in the bowel (Figure 1, N and $\mathrm{O}$ ).

Galectin-3 Expression Is Markedly Up-Regulated in the Pressure-0verloaded Myocardium and Is Localized in Both Cardiomyocytes and Noncardiomyocytes

In the normal mouse LV myocardium, galectin-3 was localized in a small number of interstitial cells with morphologic characteristics of macrophages (Figure 2A). Normal ventricular cardiomyocytes and vascular cells did not express galectin-3. In contrast, in the normal atrium, most atrial cardiomyocytes exhibited galectin-3 immunoreactivity (Figure 2B).

With the use of Mac2 immunohistochemistry we studied the time course and cellular localization of galectin-3 expression in the pressure-overloaded myocardium. The density of galectin$3^{+}$cells markedly increased after 7 and 28 days of TAC $(P<0.01$ versus control) (Figure $2 \mathrm{C})$. Remodeling of the pressure-overloaded heart was associated with profound changes in cellular localization of galectin-3. After 3 days of TAC, galectin-3 expression in the left ventricle was predominantly localized in interstitial cells with morphologic characteristics of macrophages (Figure 2D). However, after 7 to 28 days of TAC, a subset of cardiomyocytes, located in areas of fibrosis, exhibited high cytoplasmic immunoreactivity for galectin-3 (Figure 2, E and F). The specificity of Mac2 immunohistochemistry for detection and localization of galectin-3 was confirmed by the absence of immunoreactivity in sections from pressure-overloaded galectin-3 null mice (Figure 2G). In the left atrium, cardiomyocytes exhibited increased galectin-3 staining after 3 to 28 days of TAC (Figure 2, H-J). Atrial tissue from pressure-overloaded galectin-3 null mice exhibited no staining (Figure 2K), serving as a negative control.

\section{Infiltration of the Pressure-Overloaded Myocardium} with Galectin- $3^{+}$Macrophages and Myofibroblasts

To determine the cellular identity of galectin- $3^{+}$cells in the pressure-overloaded ventricle, we used dual immunofluorescence staining. Galectin-3-expressing macrophages 

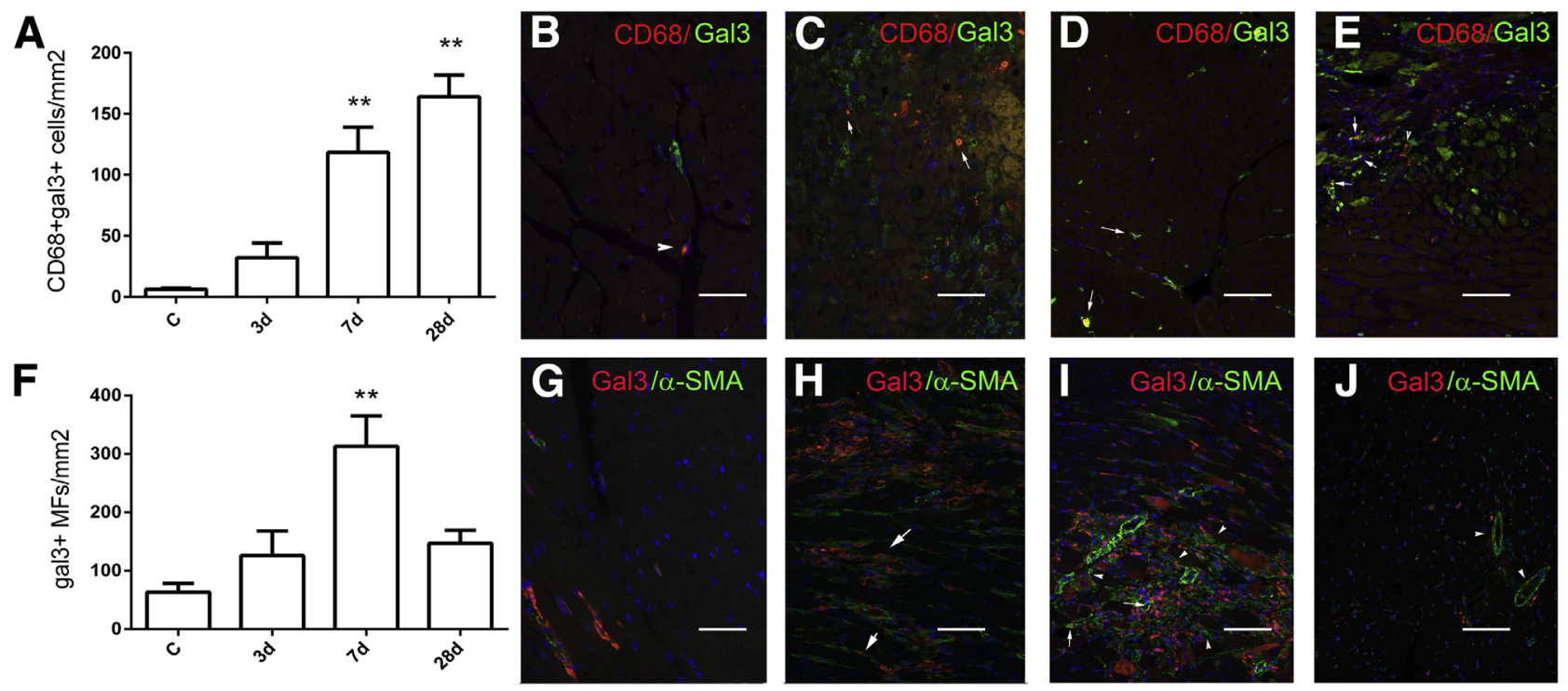

Figure 3 The pressure-overloaded myocardium is infiltrated by gal3-expressing macrophages and MFs. Dual immunofluorescence for CD68 and gal3 was used to identify gal $3^{+}$macrophages. A: The number of macrophages expressing gal3 markedly increases after 7 to 28 days of TAC. B-E: Representative images show dual staining for gal3 and CD68 in the control heart (B) and in the remodeling myocardium after 3 days (C), 7 days (D), and 28 days (E) of TAC, identifying gal $3^{+}$macrophages (arrows). Gal $3^{-}$macrophages are also noted (arrowheads). F: The density of gal $3^{+}$MFs also increases in the pressureoverloaded myocardium, peaking after 7 days of TAC. G-J: Representative images show dual staining for gal3 and $\alpha$-SMA in control myocardium (G) and in the remodeling heart after 3 days $(\mathbf{H}), 7$ days $(\mathbf{I})$, and 28 days $(\mathbf{J})$ of TAC. Gal $3^{+}$MFs were identified as interstitial spindle-shaped cells expressing $\alpha$-SMA (arrows). Many gal3 ${ }^{-}$MFs are also noted (arrowheads). J: After 28 days of TAC, the number of gal $3^{+}$MFs significantly decreases. Vascular smooth muscle cells did not exhibit galectin-3 expression (arrowheads). Data are expressed as means \pm SEM. $n=4$ to $10 .{ }^{* *} P<0.01$ versus control. C, control; gal3, galectin-3; MF, myofibroblast; TAC, transverse aortic constriction.

were identified with dual staining for Mac2 and the macrophage marker CD68. Density of galectin- $3^{+}$macrophages in the pressure-overloaded myocardium markedly increased after 7 to 28 days of TAC (Figure 3, A-E). Myofibroblasts were identified in the pressure-overloaded myocardium as $\alpha$-SMA-expressing interstitial cells, located outside the vascular media. The specificity of the $\alpha$-SMA antibody was validated with appropriate positive and negative controls (Supplemental Figure S1, F-I). The number of galectin- $3^{+}$myofibroblasts that infiltrated the pressure-overloaded myocardium markedly increased after 7 days of TAC (Figure 3, F-J).

\section{IL-1 $\beta$ and Activation of TGF- $\beta /$ Smad3 Signaling Suppress Galectin-3 Synthesis in Isolated Mouse Macrophages}

Macrophages are an important source of galectin-3. Accordingly, we examined whether the cytokines IL- $1 \beta$ and TGF- $\beta 1$, both prominently involved in cardiac remodeling, ${ }^{35,36}$ regulate galectin-3 synthesis in isolated mouse macrophages. Isolated splenic mouse macrophages exhibited high levels of galectin-3 mRNA expression. IL-1 $\beta$ and TGF- $\beta 1$ stimulation significantly reduced galectin- 3 synthesis (Figure 4A). The down-modulatory effects of IL-1 $\beta$ and TGF- $\beta 1$ were abrogated in Smad3 null macrophages, suggesting that regulation of galectin-3 synthesis in macrophages involved canonical Smad-dependent signaling. Because IL-1 $\beta$ does not directly activate Smad3 signaling, the Smad-dependent effects of IL-1 may reflect indirect actions, mediated through induction of members of the TGF- $\beta$ family.

\section{A Subset of Cardiac Fibroblasts Expresses Galectin-3}

Because a subset of myofibroblasts that infiltrated the remodeling heart expressed galectin-3, we examined whether cytokines regulate galectin-3 synthesis in isolated mouse cardiac fibroblasts cultured in collagen pads. Tcf 21 staining was used to identify fibroblasts. Approximately $10 \%$ of unstimulated $\mathrm{Tcf} 21^{+}$fibroblasts exhibited galectin-3 expression (Figure 4, B and C). IL-1 $\beta$ stimulation reduced the absolute number of galectin $3^{+}$fibroblasts (Figure 4, B-E); however, TGF- $\beta 1$ stimulation had no effect on the absolute number, or the percentage, of galectin $3^{+}$cells (Figure 4, B-F).

In the Pressure-Overloaded Myocardium, Galectin-3 Is Localized in a Subset of Cardiomyocytes

To identify and quantitate galectin- $3^{+}$cardiomyocytes, we performed dual immunofluorescence staining for WGA lectin and galectin-3. In normal left atrium, cardiomyocytes exhibited baseline galectin-3 immunoreactivity (Figure 5A) that markedly increased after 7 to 28 days of TAC (Figure 5, B and C). An atrial sample from a galectin-3 KO heart after 28 days of TAC serves as a negative control (Figure 5D). In normal mouse left ventricle, cardiomyocytes did not express galectin-3 

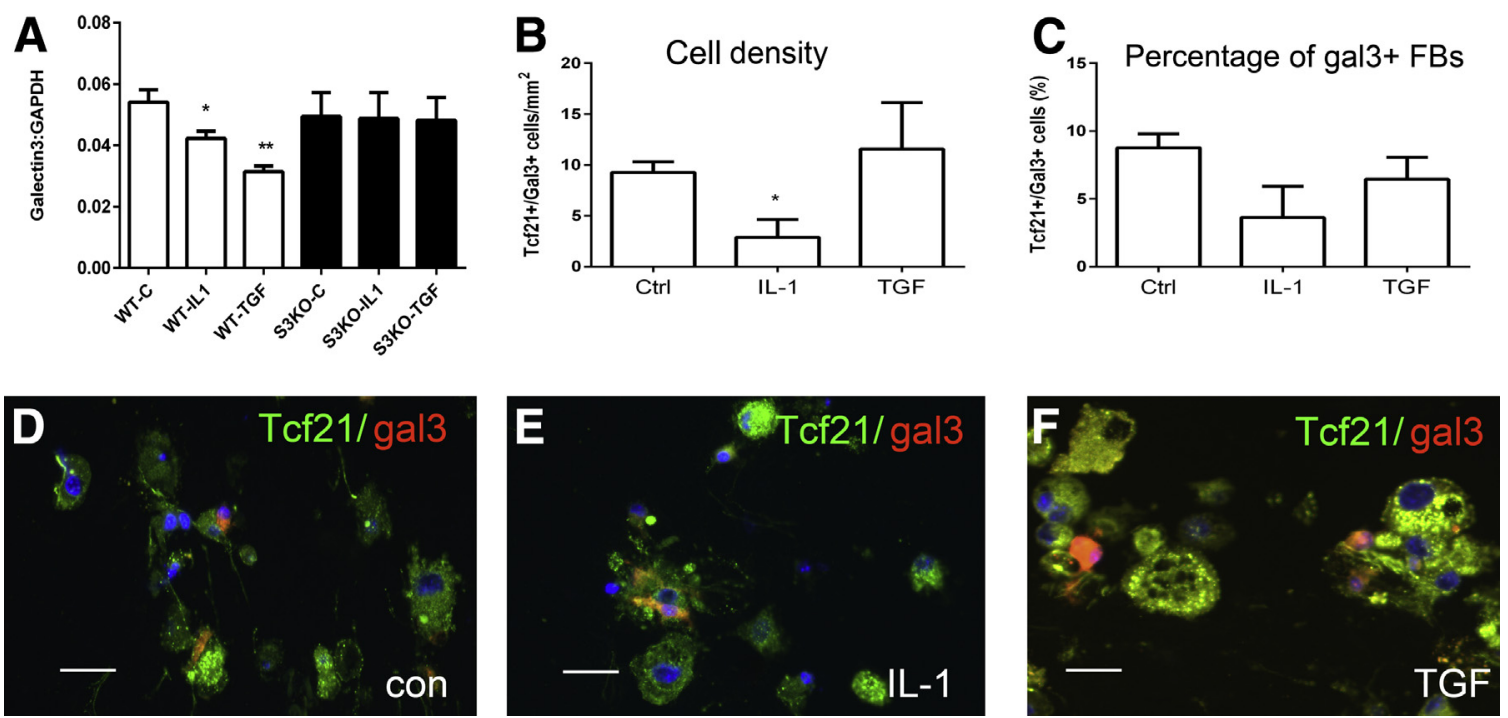

Figure 4 Regulation of gal3 in macrophages and FBs. A: The effect of cytokine stimulation on gal3 mRNA synthesis by mouse macrophages was assessed with the use of quantitative real-time PCR. Splenic mouse macrophages show high constitutive expression of gal3. IL- $1 \beta$ and TGF- $\beta 1$ suppress macrophage gal3 synthesis. IL-1 $\beta$ and TGF- $\beta 1$ do not affect gal3 synthesis in macrophages harvested from S3K0 mice, suggesting that the down-modulatory effects of the cytokines depend on Smad signaling. B and C: Dual immunofluorescence for gal33 and the FB marker Tcf21 was used to assess the effects of cytokines on gal3 expression in cardiac FBs cultured in collagen pads. A small percentage of control Tcf $21^{+}$cardiac FBs exhibit gal 3 expression. IL- $1 \beta$ decreases the density of gal3 $^{+} / \mathrm{Tcf}_{2} 1^{+}$FBs. TGF- $\beta 1$ stimulation has no effects on the number and percentage of gal3 ${ }^{+}$cells. $\mathbf{D}-\mathbf{F}$ : Representative images show gal33 expression by Tcf2 $1^{+}$FBs in control (D), IL-1 $\beta(\mathbf{E})$, and TGF- $\beta$-stimulated (F) cells. Data are expressed as means \pm SEM. $n=9$ to $12\left(\right.$ A); $n=3(\mathbf{B}-$ and $\mathbf{C})$. ${ }^{\star} P<0.05$, $* * P<0.01$ versus Ctrl. Scale bar $=20 \mu \mathrm{m}$. C, control; Ctrl, control; FB, fibroblast;gal3, galectin-3; S3K0, Smad3 knockout; Tcf21, transcription factor 21; TGF, transforming growth factor; WT, wild-type.

(Figure 5, E and F). After 7 to 28 days of TAC a significant number of cardiomyocytes exhibited galectin-3 immunoreactivity (Figure 5, G and $\mathrm{H}$ ). These cardiomyocytes were adjacent to fibrotic areas. Galectin-3 $\mathrm{KO}$ hearts served as negative controls and showed no galectin-3 staining after 28 days of TAC, despite the presence of extensive interstitial fibrosis (Figure 5I).
Myocardial Galectin-3 Expression Is Associated with Dilative Remodeling and Systolic Dysfunction, but Not with Hypertrophic Remodeling, after 28 Days of TAC

Next, we examined whether myocardial expression of galectin-3 is a marker of dysfunction and adverse remodeling in pressureoverloaded hearts. After 28 days of TAC, a strong and
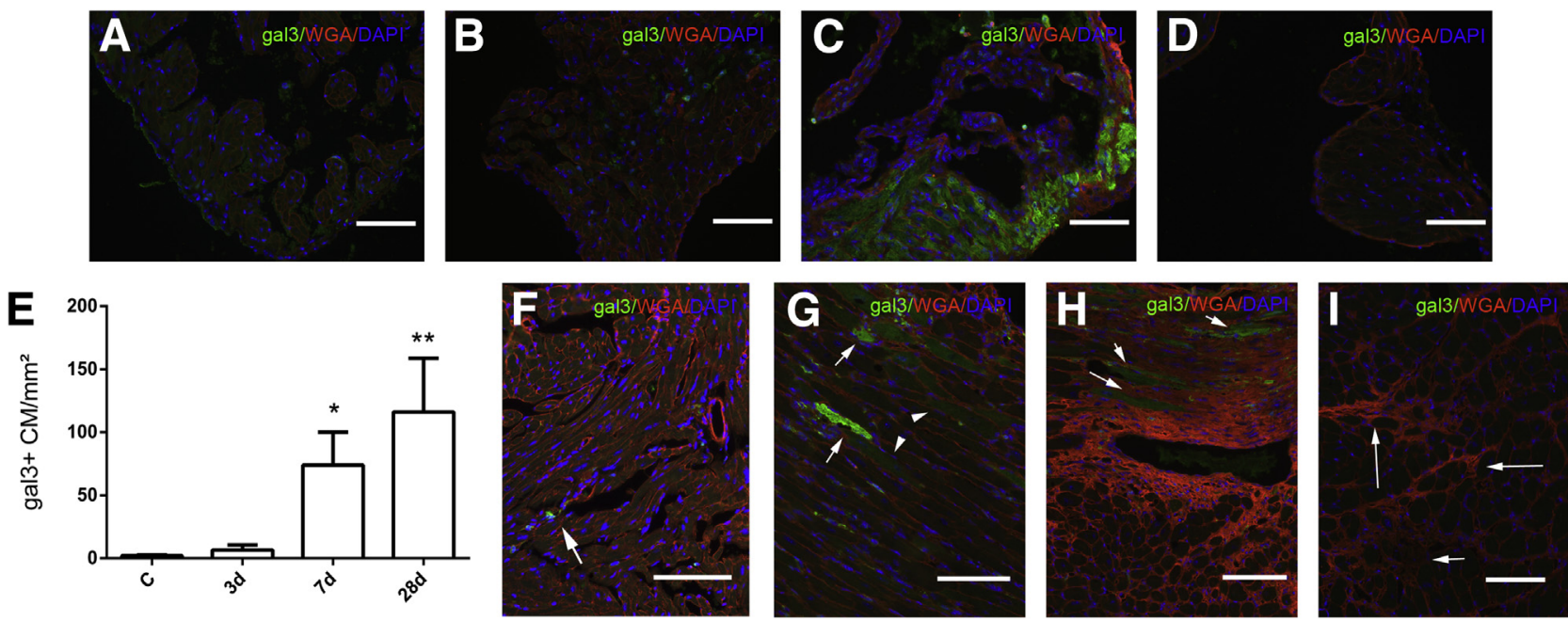

Figure 5 Gal3 localization in atrial and ventricular CMs after pressure overload. Dual immunofluorescence for gal3 (green) and WGA lectin (red) was used to identify gal $3^{+}$CMs. A-C: In normal atria, CMs show low-level gal3 immunoreactivity (A) that is markedly increased after 7 to 28 days of TAC (B and C). D: Atrial tissue from a gal3 $\mathrm{KO}$ animal after 28 days of TAC serves as a negative control. E: In the ventricular myocardium, density of gal $3^{+} \mathrm{CMs}$ markedly increases after 7 to 28 days of TAC. F: In normal ventricular myocardium, gal3 staining localizes in non-CMs (arrow). G and H: After 7 (G) and 28 days (H) of TAC, a subset of CMs adjacent to fibrotic areas exhibits strong gal3 immunoreactivity (arrows), while other CMs have weaker staining for gal3 (arrowheads). I: No gal3 immunostaining is noted in gal3 $\mathrm{KO}$ mice after 28 days of TAC, despite the presence of fibrosis (arrows). Data are expressed as means $\pm \mathrm{SEM}$. ${ }^{\star} P<0.05, * * P<0.01$ versus control. Scale bar $=60 \mu \mathrm{m}$. C, control; CM, cardiomyocyte; gal3, galectin-3; K0, knockout; TAC, transverse aortic constriction; WGA, wheat germ agglutinin. 

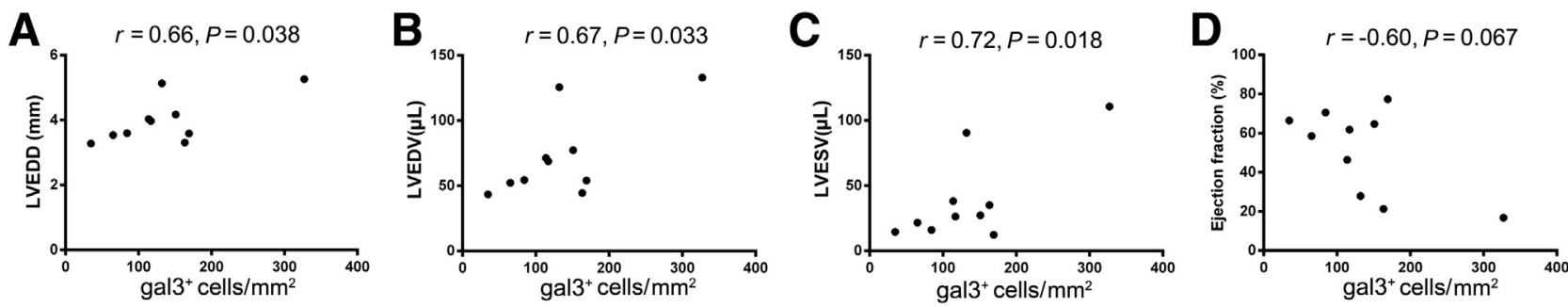

Figure 6 Ventricular density of gal3 ${ }^{+}$cells is associated with cardiac remodeling after 28 days of TAC. A-C: Correlation studies reveal statistically significant associations between the density of gal $3^{+}$cells and $\operatorname{LVEDD}(\mathbf{A}), \operatorname{LVEDV}(\mathbf{B})$, and LVESV (C). D: A trend was found toward an inverse correlation between the density of gal3 ${ }^{+}$ cells and ejection fraction that does not reach statistical significance. $n=10$. Gal3, galectin-3; LVEDD, left ventricular end-diastolic diameter; LVEDV, left ventricular enddiastolic volume; LVESD, left ventricular end-systolic diameter; LVESV, left ventricular end-systolic volume; TAC, transverse aortic constriction.

statistically significant positive correlation was found between the density of galectin- $3^{+}$cells and ventricular dimensions (Figure 6, A-C). In addition, a trend toward an inverse correlation was found between the density of galectin- $3^{+}$cells and ejection fraction (Figure 6D). In contrast, no association was found between the density of galectin- $3^{+}$cells and LV mass $(r=0.12, P=0.73)$.

\section{Galectin-3 Expression in Cardiomyocytes, but Not in} Macrophages, Is Associated with Dilative Remodeling and Systolic Dysfunction

We then examined the association between cell-specific galectin-3 localization and cardiac remodeling after pressure overload. After 28 days of TAC, a strong correlation was found between the density of galectin- $3^{+}$ cardiomyocytes and ventricular dimensions (Figure 7, $\mathrm{A}-\mathrm{C})$. We also noted a trend toward an inverse correlation between the density of galectin- $3^{+}$cardiomyocytes and ejection fraction (Figure 7D). Trends were found for weaker correlations between the density of galectin- $3^{+}$ myofibroblasts and ventricular dimensions (Figure 7, $\mathrm{E}-\mathrm{G}$ ) and a trend for an inverse correlation between the number of galectin-3-expressing myofibroblasts and ejection fraction (Figure $7 \mathrm{H}$ ). In contrast, no association was found between the density of galectin- $3^{+}$ macrophages and the end points that reflect cardiac remodeling, dysfunction, and hypertrophy (galectin- $3^{+}$ macrophages and LV end-diastolic diameter: $r=0.10$, $P=0.78$; galectin- $3^{+}$macrophages and LVEDV: $r=0.06, P=0.86$; galectin- $3^{+}$macrophages and LV end-systolic volume: $r=-0.06, P=0.88$; galectin- $3^{+}$ macrophages and ejection fraction: $r=0.25, P=0.53$; gal $^{+}$macrophages and LV mass: $r=-0.35$, $P=0.32)$.

\section{Galectin-3 Loss Does Not Affect Survival after Pressure Overload}

To examine whether galectin-3 plays a critical role in remodeling of the pressure-overloaded heart, we compared ventricular dimensions and cardiac function between WT
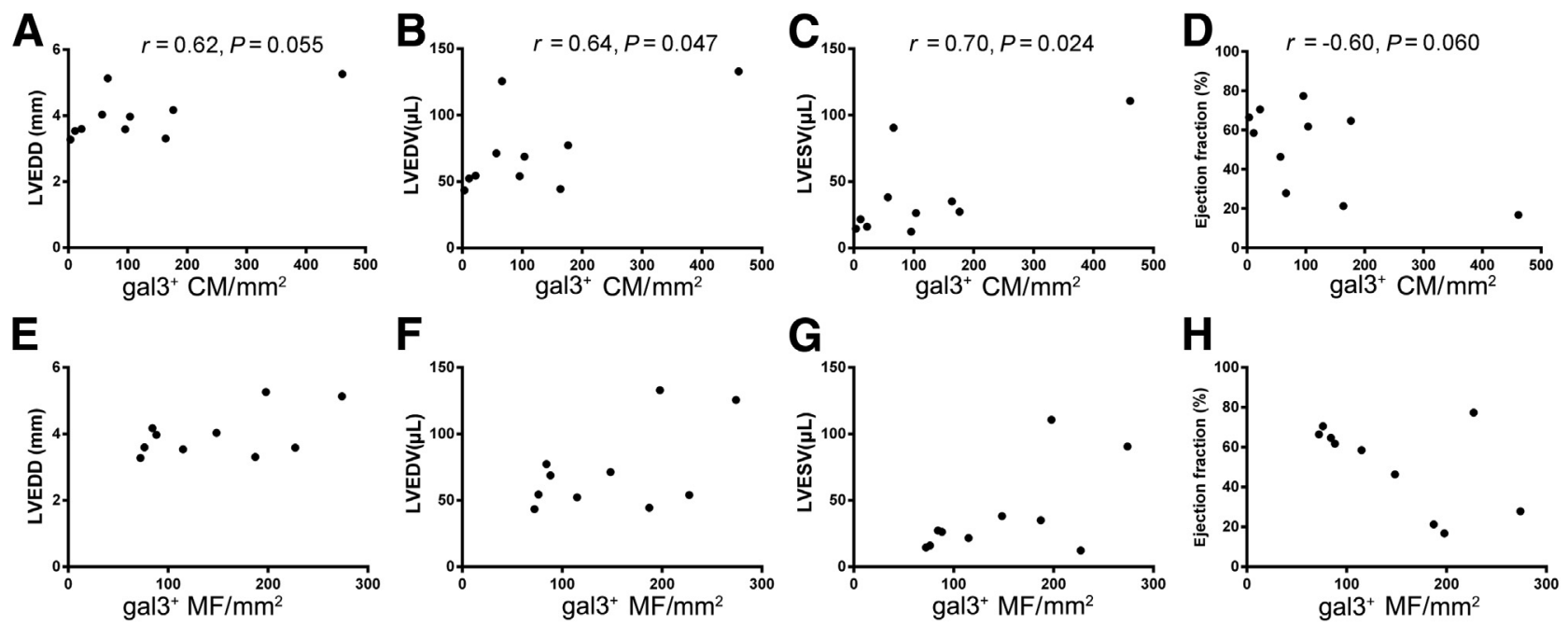

Figure 7 Ventricular density of gal3 ${ }^{+}$CMs is associated with adverse remodeling after 28 days of TAC. A-D: A statistically significant correlation was found between the density of gal3 ${ }^{+}$CMs and left ventricular volumes (B, LVEDV; C, LVESV). Moreover, a trend was found toward a positive correlation between the density of gal $3^{+}$CMs and $\operatorname{LVEDD}(\mathbf{A})$ and a trend was found toward a negative correlation between the density of gal $3^{+}$CMs and ejection fraction (D). E-H: Associations between the density of gal $3^{+}$MFs and remodeling-associated variables are weaker and do not reach statistical significance. No significant associations were found between the density of gal $3^{+}$macrophages and cardiac remodeling. $n=10$. CM, cardiomyocyte; gal3, galectin-3; LVEDD, left ventricular end-diastolic diameter; LVEDV, left ventricular end-diastolic volume; LVESV, left ventricular end-systolic volume; MF, myofibroblast; TAC, transverse aortic constriction. 

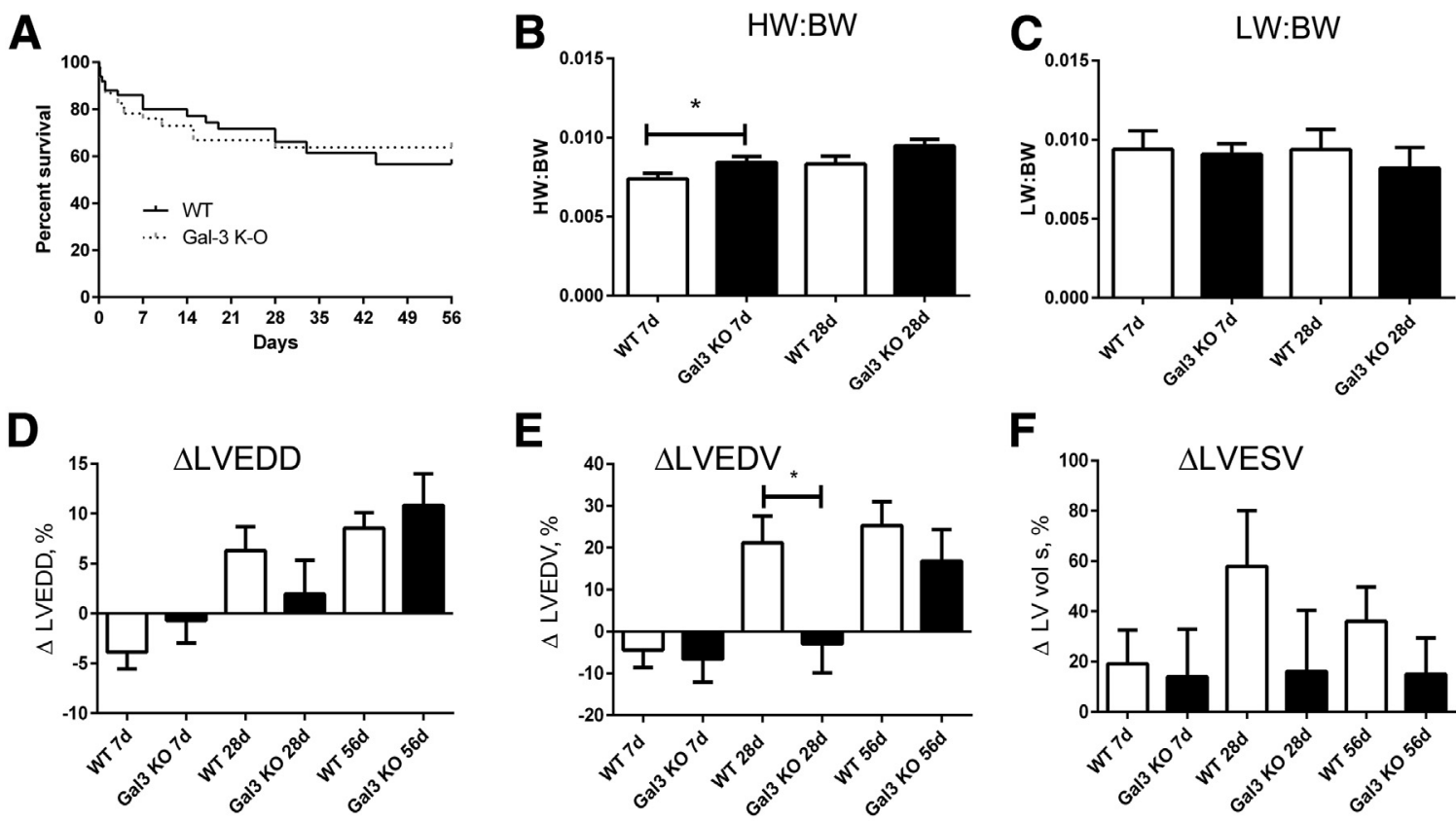

E
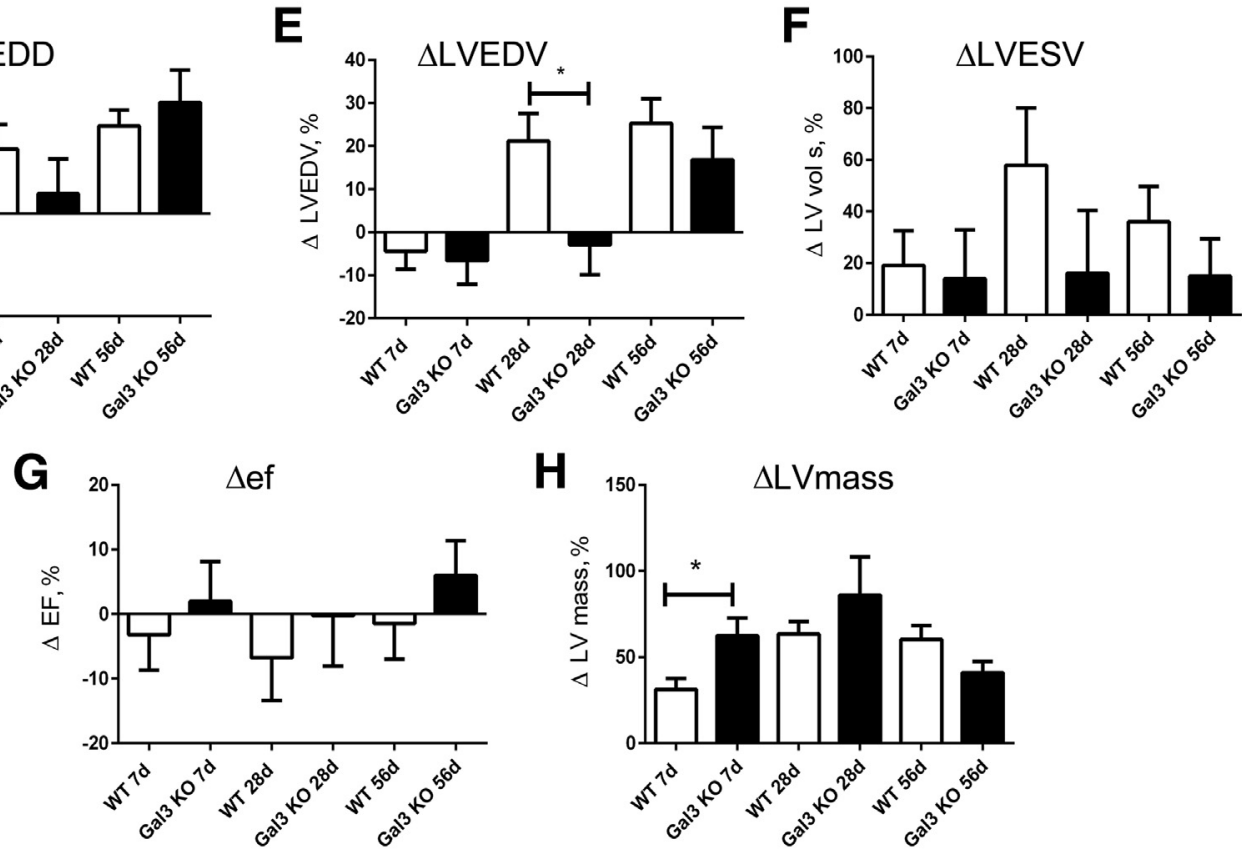

Figure 8 Gak3 deficiency accelerates hypertrophy and delays chamber dilation without affecting survival and systolic dysfunction after pressure overload. A: WT and gal3 $\mathrm{KO}$ mice have comparable survival curves after pressure overload. B: The HW/BW is significantly higher in gal3 $\mathrm{KO}$ mice after 7 days of TAC. A trend was found toward increased HW/BW after 28 days of TAC that does not reach statistical significance. C: LW/BW is comparable between groups. D: Gal3 loss does not affect $\triangle$ LVEDD after 7 to 56 days of TAC. E: $\triangle$ LVEDV is significantly lower in gal3 K0 mice after 28 days of TAC. $\mathbf{F}$ and $\mathbf{G}$ : No significant differences were found in $\triangle$ LVESV and $\triangle$ EF. H: After 7 days of TAC, gal3 $\mathrm{KO}$ mice exhibit an accentuated increase in LV mass, suggesting accelerated hypertrophy. However, after 28 days of TAC LV mass is comparable between WT and gal3 K0 mice. Data are expressed as means \pm SEM. $n=50$ WT, $n=46 \mathrm{KO}(\mathbf{A}) ; n=10$ to 11 per group (B); $n=37$ WT mice, $n=30 \mathrm{KO}$ mice at 7 days $(\mathbf{H}) ; n=25$ WT mice, $n=18 \mathrm{KO}$ mice at 28 days $(\mathbf{H}) ; n=13$ WT mice, $n=10$ mice at 56 days $(\mathbf{H})$. ${ }^{\star} P<0.05$ versus WT. gal3, galectin-3; HW/BW, heart weight-to-body weight ratio; K0, knockout; LV, left ventricular; LW/BW, lung weight-to-body weight ratio; TAC, transverse aortic constriction; WT, wild-type; $\Delta \mathrm{EF}$, change in ejection fraction; $\Delta \mathrm{LVEDD}$, change in left ventricular end-diastolic diameter; $\Delta \mathrm{LVEDV}$, change in left ventricular enddiastolic volume; $\triangle$ LVESD, change in left ventricular end-systolic diameter; $\Delta$ LVESV, change in left ventricular end-systolic volume.

and galectin-3 KO mice undergoing TAC protocols. WT and galectin-3 mice had comparable left-to-right carotid flow ratios after TAC (WT, $0.14 \pm 0.01 ; \mathrm{KO}, 0.15 \pm 0.01)$ suggesting comparable pressure load between groups. Survival curves were comparable between WT and galectin-3 KO mice (Figure 8A).

\section{Galectin-3 Loss Accelerates Hypertrophy and Delays} Chamber Dilation, but Does Not Significantly Affect Function and Remodeling of the Pressure-Overloaded Heart

Heart weight-to-body weight ratio was significantly higher in galectin-3 KO mice after 7 days of TAC (Figure 8, B and C). At the 28-day time point the difference in LV mass between WT and KO mice was no longer significant (Figure 8, B and C). Lung weight/body weight ratio was comparable between groups (Figure 8, D and E). Echocardiographic analysis showed that WT and galectin-3 KO mice had delayed chamber dilation after pressure overload, evidenced by a markedly lower change in LVEDV after 28 days of TAC (Figure 8, D and E). However, changes in LV end-systolic volume and in ejection fraction were comparable between groups (Figure 8, F and G). Galectin-3 KO mice had an accentuated increase in LV mass after 7 days of TAC; however, at the 28-day and 56-day time points, no differences were found between the groups (Figure $8 \mathrm{H}$ ). Sex-specific analysis showed that galectin-3 loss delayed chamber dilation in female mice but not in male mice undergoing pressure overload protocols (Figure 9). 

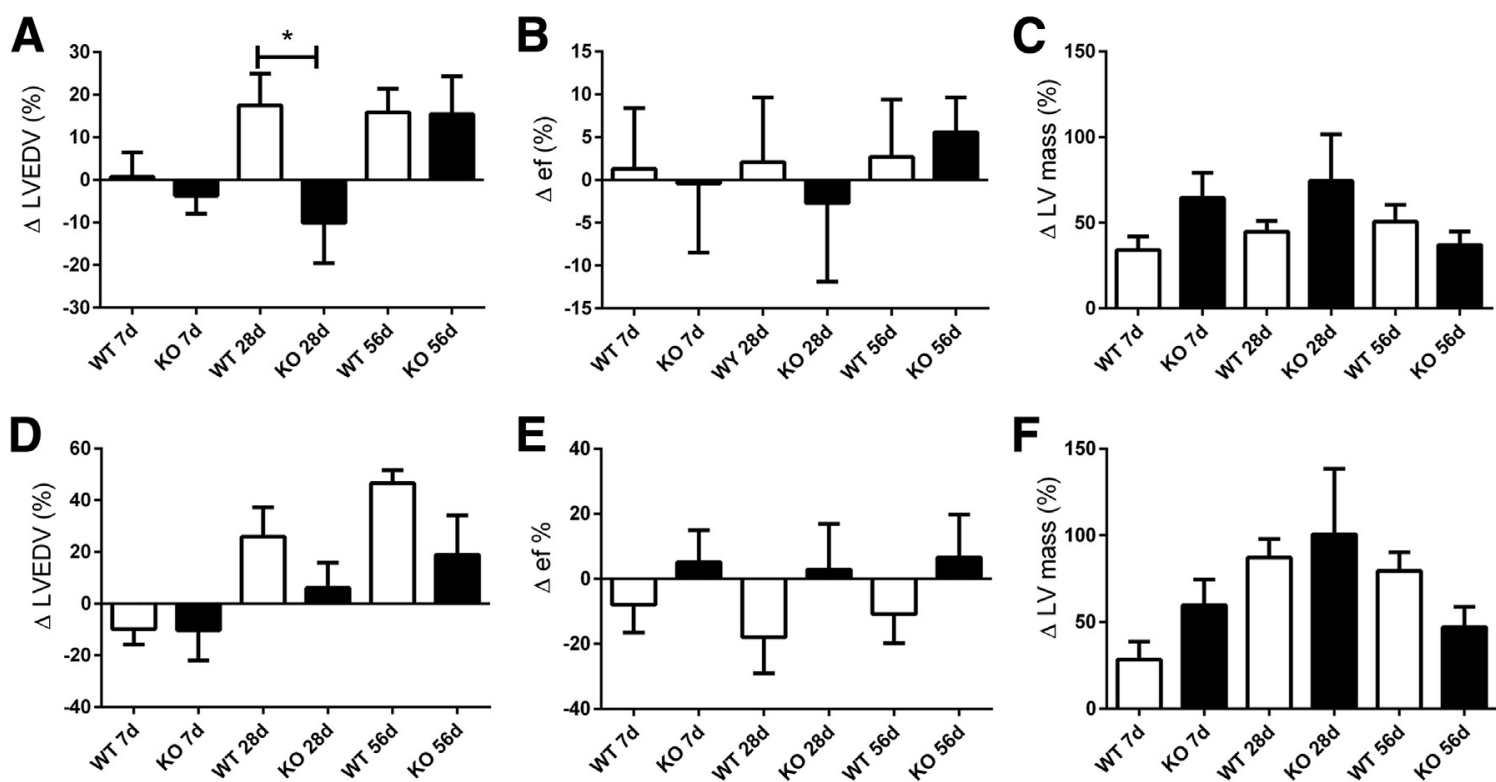

Figure 9 Sex-specific effects of galectin-3 absence on the pressure-overloaded heart. Galectin-3 loss delays dilation in female (A), but not in male (D) mice, increasing the $\triangle$ LVEDV after 28 days of TAC. Galectin-3 loss does not affect $\Delta$ ef in female (B) or male (E) mice. In both female (C) and male (F) mice, galectin-3 loss is associated with a trend toward increased $\Delta$ LV mass after 7 days of TAC that does not reach statistical significance. Variables were measured by echocardiography. Data are expressed as means \pm SEM. $n=19$ female WT mice, $n=17$ female K0 mice at 7 days; $n=14$ WT female mice, $n=10$ female K0 mice at 28 days; $n=9$ female WT mice, $n=6$ female K0 mice at 56 days; $n=18$ male WT mice, $n=13$ male K0 mice at 7 days; $n=11$ male WT mice, $n=8$ male K0 mice at 28 days; $n=4$ male WT mice, $n=4$ male K0 mice at 56 days. ${ }^{*} P<0.05$. K0, knockout; TAC, transverse aortic constriction; WT, wildtype; $\Delta$ ef, change in ejection fraction; $\Delta$ LV, change in left ventricular; $\triangle$ LVEDV, change in left ventricular end-diastolic volume; $\Delta$ LVESD, change in left ventricular end-systolic diameter; $\Delta$ LVESV, change in left ventricular end-systolic volume.

\section{Galectin-3 Loss Does Not Significantly Affect Diastolic Dysfunction, Collagen Content, and Cardiomyocyte Hypertrophy}

We used mitral inflow Doppler echocardiography and tissue Doppler imaging to examine whether galectin-3 loss affects diastolic function in pressure-overloaded hearts. Both WT and galectin-3 KO mice developed tachycardia (Figure 10A) and had increased peak E and A velocities (Figure 10, B and $\mathrm{C})$, associated with a late reduction in the $\mathrm{E} / \mathrm{A}$ ratio (Figure 10D). Tissue Doppler imaging showed that both WT and galectin-3 KO mice had increased $\mathrm{E} / \mathrm{E}^{\prime}$ ratio and reduced $\mathrm{E}^{\prime} / \mathrm{A}^{\prime}$ ratio (Figure 10, $\mathrm{E}$ and $\mathrm{F}$ ). These findings are consistent with diastolic dysfunction. No significant differences were found in variables that reflect diastolic function between WT and galectin-3 KO mice.

To examine the effects of galectin- 3 on cardiac fibrosis after pressure overload, we performed Sirius red staining in WT and galectin-3 KO hearts after 28 days of TAC (Figure 10G). Quantitative analysis of collagen content found no significant differences in collagen content between WT and galectin-3 null hearts (Figure 10, H-J). Moreover, we used WGA staining to assess the effects of galectin-3 on cardiomyocyte size (Figure 10K) after pressure overload. After 28 days of TAC, cardiomyocyte cross-sectional area was comparable between WT and KO mice (Figure 10, L-N). Sex-specific analysis showed no significant effects of galectin-3 loss on male or female mice (Figure 10, I, J, M and N).

\section{Discussion}

Our study reports several novel observations. First, we found that, despite its potential significance as a biomarker in patients with heart failure, galectin-3 plays a noncritical role in the pathogenesis of cardiac remodeling after pressure overload. Global loss of galectin-3 accelerated cardiac hypertrophy and delayed dilative remodeling but did not affect survival, systolic and diastolic dysfunction, fibrosis, and late geometric and functional remodeling of the pressureoverloaded heart. Second, we demonstrate for the first time that galectin-3 is not exclusively localized in macrophages that infiltrate the pressure-overloaded myocardium. Atrial and ventricular cardiomyocytes and activated myofibroblasts exhibit galectin-3 immunoreactivity in the pressureoverloaded mouse heart. Cardiomyocyte-specific galectin-3 localization is associated with adverse remodeling and dysfunction after pressure overload.

In the Pressure-0verloaded Myocardium, Galectin-3 Is Localized in Activated Macrophages, Cardiomyocytes, and Myofibroblasts

Galectin-3 has been identified as a galactose-specific lectin that is abundantly produced by thioglycollate-activated macrophages. ${ }^{37}$ In mouse models of tissue injury, galectin3 immunohistochemistry has been extensively used for identification of activated macrophages. ${ }^{38-40}$ However, 
A

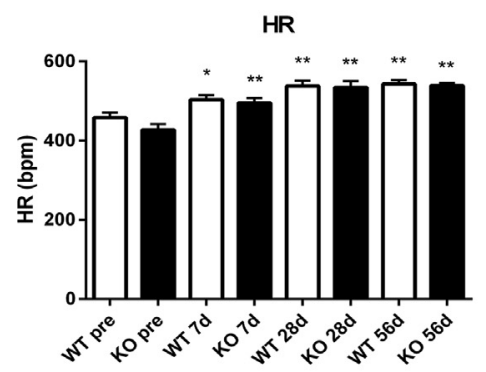

D

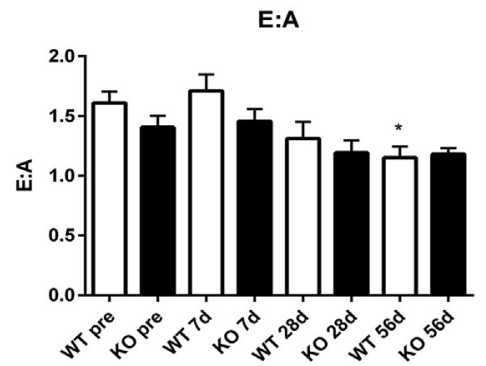

B

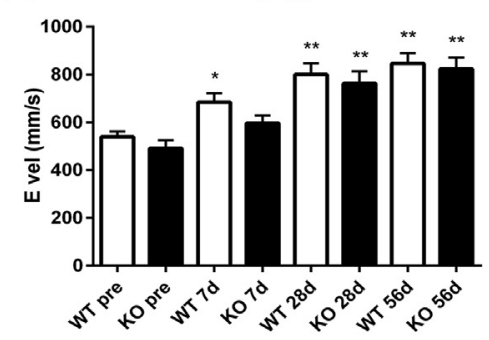

E

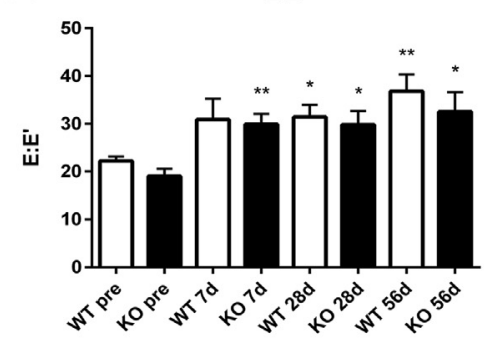

C

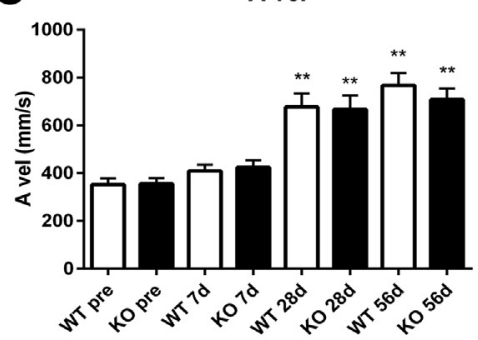

F

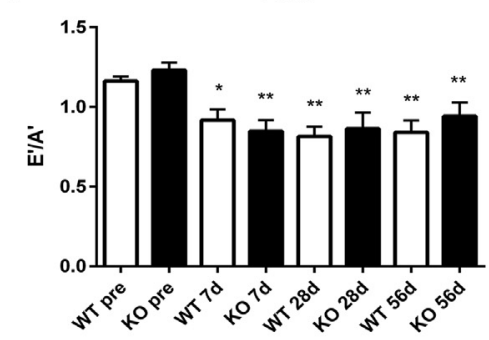

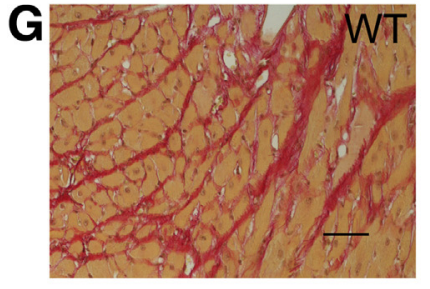

K

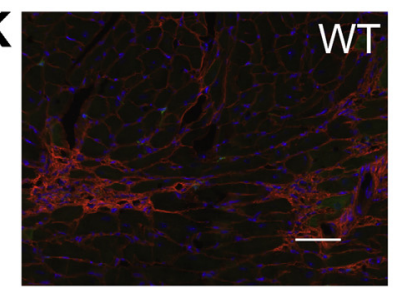

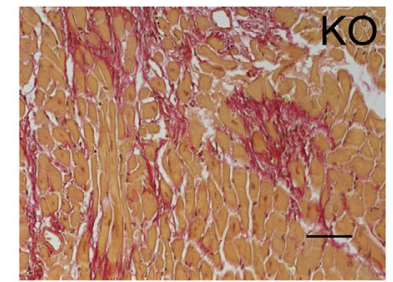
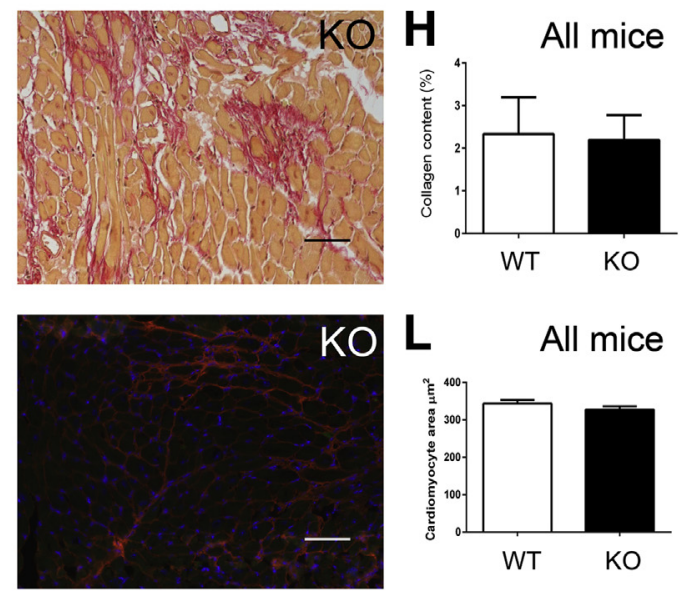
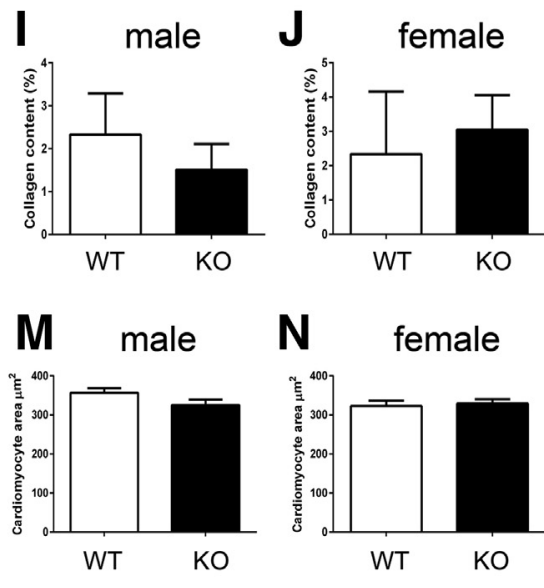

Figure 10 Galectin-3 loss does not affect diastolic dysfunction, fibrosis, and cardiomyocyte hypertrophy in the pressure-overloaded myocardium. A-F: Doppler echocardiography and tissue Doppler imaging shows that galectin-3 absence does not affect diastolic function. TAC increases (A) and peak E (B) and A (C) velocities in both WT and galectin-3 $\mathrm{KO}$ mice. The E/A ratio is significantly reduced in WT mice after 56 days of TAC (D). The E/E' ratio, a sensitive indicator of diastolic dysfunction, increases after TAC in both WT and galectin-3 $\mathrm{KO}$ mice $(\mathbf{E})$, whereas the $\mathrm{E}^{\prime} / \mathrm{A}^{\prime}$ ratio is reduced (F). Galectin-3 loss has no effects on indicators of diastolic dysfunction. G-J: Sirius red staining was used to label collagen fibers in WT and galectin-3 KO hearts after 28 days of TAC. No statistically significant difference was noted between WT and K0 mice $(\mathbf{H})$ in both male and female groups. $\mathbf{K}-\mathbf{N}$ : Cardiomyocyte size was assessed in sections stained for WGA lectin. Cardiomyocyte size was comparable in WT and galectin-3 K0 mice after 28 days of TAC (L), in both male and female mice. Data are expressed as means \pm SEM. $n=10$ to 16 per group $(\mathbf{A}-\mathbf{F}) ; n=9$ to 10 per group $(\mathbf{K}-\mathbf{N})$. ${ }^{*} P<0.05,{ }^{*} P<0.01$ versus corresponding pre. Scale bar $=60$ $\mu \mathrm{m}$. Bpm, beats per minute; HR, heart rate; KO, knockout; TAC, transverse aortic constriction; WT, wild-type.

galectin-3 expression is not limited to cells of the macrophage lineage. Although in many normal mouse tissues (such as the spleen, liver, and lung), galectin-3 immunoreactivity is exclusively localized in macrophages, epithelial cells in the kidney and the intestine also express large amounts of galectin-3 (Figure 1).

Our findings suggest that in the myocardium, galectin-3 localization is not limited to macrophages. In normal adult mouse hearts, atrial but not ventricular cardiomyocytes exhibited constitutive galectin-3 immunoreactivity (Figure 2). We observed marked up-regulation of galectin-3 in the pressure-overloaded heart, confirming similar findings in other models of hypertrophic cardiac remodeling. ${ }^{21}$ Transgenic rats overexpressing the mouse renin gene (Ren-2) exhibit severe hypertension and develop marked cardiac hypertrophy followed by decompensation and overt heart failure. ${ }^{41}$ Cardiac remodeling in Ren-2 rats is associated with marked up-regulation of galectin-3 expression that is predominantly localized in infiltrating macrophages. ${ }^{21}$ As expected, in our model of TAC-induced cardiac pressure overload, myocardial remodeling was also associated with infiltration of numerous galectin- $3^{+}$macrophages (Figure 3). Because cytokine stimulation has been previously implicated in galectin-3 up-regulation in macrophages, ${ }^{42,43}$ we examined 
whether IL-1 $\beta$ and TGF- $\beta 1$, cytokines critically involved in cardiac remodeling, ${ }^{35,36}$ regulate macrophage galectin-3 synthesis. Unstimulated splenic macrophages expressed high levels of galectin-3; however, both IL-1 $\beta$ and activation of the TGF- $\beta 1 /$ Smad pathway suppressed galectin-3 synthesis (Figure 4). Smad3 absence abrogated the downmodulatory effects of IL-1 $\beta$, suggesting that the actions of the cytokine may involve indirect stimulation of a Smaddependent TGF- $\beta$ cascade. Macrophages are highly heterogeneous and exhibit distinct properties, depending on the tissue of origin $^{44}$; thus, whether responses of splenic macrophages to cytokine stimulation are relevant in cardiac remodeling is unclear.

After 7 days of pressure overload, a subset of myofibroblasts also expressed galectin-3. Expression of galectin-3 by fibroblasts has been previously reported. ${ }^{45,46}$ In mouse $3 \mathrm{~T} 3$ fibroblasts and in the LG1 strain of human diploid fibroblasts, galectin-3 expression was associated with cell proliferation. ${ }^{45,46}$ Our experiments showed that approximately $10 \%$ of unstimulated cardiac fibroblasts expressed galectin-3 when cultured in collagen pads (Figure 4). IL-1 $\beta$ and TGF- $\beta$ stimulation did not increase galectin-3 expression in cardiac fibroblasts (Figure 4), suggesting that the increased number of galectin-3 myofibroblasts in the remodeling myocardium may not directly result from cytokine stimulation but may reflect the increased infiltration of the pressure-overloaded myocardium with abundant activated fibroblasts.

Surprisingly, after 7 to 28 days of pressure overload, intense galectin-3 immunoreactivity was localized in a subset of ventricular cardiomyocytes. Galectin-3 expression by cardiomyocytes has not been previously reported in vivo or in vitro. We demonstrated the specificity of Mac2 immunohistochemistry in detection of galectin-3 by showing absence of staining in pressure-overloaded galectin-3 KO hearts (Figure 5). Moreover, the clear cytoplasmic localization of galectin-3 in a subset of ventricular cardiomyocytes in fibrotic myocardial areas (Figure 5) suggests that immunoreactivity does not result from binding of the protein to the cell surface. Whether galectin-3 localization in cardiomyocytes of the pressure-overloaded heart indicates active synthesis or reflects the transfer of protein produced by other cell types (such as macrophages or myofibroblasts) remains unclear.

\section{Galectin-3 in Cardiomyocytes Is Associated with Dysfunction and Adverse Remodeling}

The significance of galectin-3 expression in regulation of the biological properties of cardiomyocytes remains unclear. Our findings indicated that a high density of galectin- $3^{+}$ cardiomyocytes was associated with adverse remodeling and dysfunction in pressure-overloaded hearts. The absence of major effects of galectin-3 loss in cardiac remodeling suggests that cardiomyocyte-specific galectin-3 is unlikely to play a crucial role in cardiomyocyte responses to pressure overload. Thus, expression of galectin-3 in cardiomyocytes may represent an epiphenomenon that reflects another injurious cellular process. Protective effects of galectin-3 in apoptotic responses have been extensively reported ${ }^{47,48}$; whether expression of galectin-3 by selected cardiomyocytes serves a protective role against apoptosis is unclear. However, the absence of significant effects of galectin-3 loss on cardiac function does not support a crucial effect of galectin-3 in protecting cardiomyocytes in the pressure-overloaded heart.

\section{Does Galectin-3 Play a Role in Cardiac Remodeling?}

In vitro and in vivo studies have suggested an important role for galectin-3 in regulation of inflammatory and fibrotic responses. ${ }^{7,49}$ Studies on the role of galectin-3 in inflammation have produced contradictory results, likely reflecting the context-dependent actions of intracellular and matrixbound galectin-3 in a variety of cell types. In a model of thioglycollate-induced peritonitis, galectin-3 KO animals exhibited attenuated peritoneal inflammation, and loss of galectin-3 increased apoptosis of proinflammatory macrophages and decreased NF- $\kappa \mathrm{B}$ signaling. ${ }^{50}$ In contrast, in a model of endotoxemia, galectin-3 loss was associated with an accentuated inflammatory response, suggesting that it may serve as a negative regulator of endotoxin-induced proinflammatory activation. ${ }^{10}$

Galectin-3 expression is consistently induced in models of fibrosis; its up-regulation is associated with activation of a profibrotic program. In experimental hepatic fibrosis, galectin-3 loss inhibited myofibroblast activation by attenuating TGF- $\beta$-mediated responses. ${ }^{11}$ Profibrotic actions of galectin-3 were also reported in models of renal ${ }^{12}$ and pulmonary ${ }^{51}$ injury. In models of fibrotic cardiomyopathy induced by pressure overload, $\mathrm{Yu}$ et $\mathrm{al}^{22}$ suggested that galectin-3 plays an important role in the pathogenesis of fibrosis and dysfunction. Moreover, in a model of aldosterone-induced cardiac fibrosis, galectin-3 was critically involved in fibroblast activation. ${ }^{23,52}$ Our findings, based on a higher number of mice, do not support these published observations. With the use of the same loss-offunction model, we found no significant effects of galectin-3 loss on cardiac dysfunction and fibrosis in the pressure-overloaded heart. The disparate findings may reflect sex-specific effects: in the study by $\mathrm{Yu}$ et al, ${ }^{22}$ and in the investigations on the model of aldosterone-induced cardiomyopathy only male animals were studied. Sexspecific analysis of the echocardiographic data showed that galectin-3 loss delayed dilation of the pressureoverloaded heart in female mice (Figure 9) but did not suggest protective actions of galectin-3 in male animals. Moreover, differences in the severity of the pressure load and exclusive focus on a single time point may account for the distinct observations. In our investigation, assessment of the carotid flow ratio ensures comparable pressure gradients between WT and KO mice. Moreover, we performed systematic analysis of cardiac remodeling and function after 7,28 , and 56 days of TAC to investigate the consequences 
of galectin-3 loss in the transition from hypertrophic to dilative remodeling.

Our observations suggested that galectin-3 loss may delay the hypertrophic response after pressure overload. The findings may suggest direct activation of a hypertrophic program in cardiomyocytes by galectin-3 or may reflect galectin-3-mediated modulation of macrophages toward a phenotype that promotes hypertrophy. Note that the absence of significant differences in function and geometry between WT and galectin-3 KO mice may reflect competing actions of galectin-3 on various cell types involved in cardiac remodeling.

\section{Conclusions}

Despite its role as a biomarker that mirrors the progression and severity of heart failure, ${ }^{53}$ galectin-3 does not play a crucial role in the pathogenesis of the fibrotic cardiomyopathy associated with pressure overload. Useful and reliable biomarkers of cardiac remodeling do not necessarily serve a key biological function, but they may be released after stress, reflecting another injurious process. Galectin-3 localization in atrial and ventricular cardiomyocytes and expression by infiltrating myofibroblasts may be an indicator of myocardial injury and fibrosis, thus explaining the association between galectin-3 levels and adverse prognosis.

\section{Supplemental Data}

Supplemental material for this article can be found at http://dx.doi.org/10.1016/j.ajpath.2015.12.017.

\section{References}

1. Sato S, Hughes RC: Regulation of secretion and surface expression of Mac-2, a galactoside-binding protein of macrophages. J Biol Chem 1994, 269:4424-4430

2. Liu FT, Hsu DK, Zuberi RI, Kuwabara I, Chi EY, Henderson WR Jr: Expression and function of galectin-3, a beta-galactoside-binding lectin, in human monocytes and macrophages. Am J Pathol 1995, 147:1016-1028

3. Kaltner H, Seyrek K, Heck A, Sinowatz F, Gabius HJ: Galectin-1 and galectin-3 in fetal development of bovine respiratory and digestive tracts. Comparison of cell type-specific expression profiles and subcellular localization. Cell Tissue Res 2002, 307:35-46

4. Neidhart M, Zaucke F, von Knoch R, Jungel A, Michel BA, Gay RE, Gay S: Galectin-3 is induced in rheumatoid arthritis synovial fibroblasts after adhesion to cartilage oligomeric matrix protein. Ann Rheum Dis 2005, 64:419-424

5. Rao SP, Wang Z, Zuberi RI, Sikora L, Bahaie NS, Zuraw BL, Liu FT, Sriramarao P: Galectin-3 functions as an adhesion molecule to support eosinophil rolling and adhesion under conditions of flow. J Immunol 2007, 179:7800-7807

6. Lotan R, Belloni PN, Tressler RJ, Lotan D, Xu XC, Nicolson GL: Expression of galectins on microvessel endothelial cells and their involvement in tumour cell adhesion. Glycoconj J 1994, 11:462-468

7. Henderson NC, Sethi T: The regulation of inflammation by galectin3. Immunol Rev 2009, 230:160-171
8. MacKinnon AC, Farnworth SL, Hodkinson PS, Henderson NC, Atkinson KM, Leffler H, Nilsson UJ, Haslett C, Forbes SJ, Sethi T: Regulation of alternative macrophage activation by galectin-3. J Immunol 2008, 180:2650-2658

9. Dragomir AC, Sun R, Choi H, Laskin JD, Laskin DL: Role of galectin-3 in classical and alternative macrophage activation in the liver following acetaminophen intoxication. J Immunol 2012, 189: $5934-5941$

10. Li Y, Komai-Koma M, Gilchrist DS, Hsu DK, Liu FT, Springall T, $\mathrm{Xu} \mathrm{D:} \mathrm{Galectin-3}$ is a negative regulator of lipopolysaccharidemediated inflammation. J Immunol 2008, 181:2781-2789

11. Henderson NC, Mackinnon AC, Farnworth SL, Poirier F, Russo FP, Iredale JP, Haslett C, Simpson KJ, Sethi T: Galectin-3 regulates myofibroblast activation and hepatic fibrosis. Proc Natl Acad Sci U S A 2006, 103:5060-5065

12. Henderson NC, Mackinnon AC, Farnworth SL, Kipari T, Haslett C, Iredale JP, Liu FT, Hughes J, Sethi T: Galectin-3 expression and secretion links macrophages to the promotion of renal fibrosis. Am J Pathol 2008, 172:288-298

13. Calvier L, Miana M, Reboul P, Cachofeiro V, Martinez-Martinez E, de Boer RA, Poirier F, Lacolley P, Zannad F, Rossignol P, LopezAndres N: Galectin-3 mediates aldosterone-induced vascular fibrosis. Arterioscler Thromb Vasc Biol 2013, 33:67-75

14. Inohara H, Akahani S, Raz A: Galectin-3 stimulates cell proliferation. Exp Cell Res 1998, 245:294-302

15. Seropian IM, Cerliani JP, Toldo S, Van Tassell BW, Ilarregui JM, Gonzalez GE, Matoso M, Salloum FN, Melchior R, Gelpi RJ, Stupirski JC, Benatar A, Gomez KA, Morales C, Abbate A, Rabinovich GA: Galectin-1 controls cardiac inflammation and ventricular remodeling during acute myocardial infarction. Am J Pathol 2013, 182:29-40

16. de Boer RA, Voors AA, Muntendam P, van Gilst WH, van Veldhuisen DJ: Galectin-3: a novel mediator of heart failure development and progression. Eur J Heart Fail 2009, 11:811-817

17. van Kimmenade RR, Januzzi JL Jr, Ellinor PT, Sharma UC, Bakker JA, Low AF, Martinez A, Crijns HJ, MacRae CA, Menheere PP, Pinto YM: Utility of amino-terminal pro-brain natriuretic peptide, galectin-3, and apelin for the evaluation of patients with acute heart failure. J Am Coll Cardiol 2006, 48:1217-1224

18. Felker GM, Fiuzat M, Shaw LK, Clare R, Whellan DJ, Bettari L, Shirolkar SC, Donahue M, Kitzman DW, Zannad F, Pina IL, O'Connor CM: Galectin-3 in ambulatory patients with heart failure: results from the HF-ACTION study. Circ Heart Fail 2012, 5:72-78

19. Ho JE, Liu C, Lyass A, Courchesne P, Pencina MJ, Vasan RS, Larson MG, Levy D: Galectin-3, a marker of cardiac fibrosis, predicts incident heart failure in the community. J Am Coll Cardiol 2012, 60: $1249-1256$

20. Lok DJ, Lok SI, Bruggink-Andre de la Porte PW, Badings E, Lipsic E, van Wijngaarden J, de Boer RA, van Veldhuisen DJ, van der Meer P: Galectin-3 is an independent marker for ventricular remodeling and mortality in patients with chronic heart failure. Clin Res Cardiol 2013, 102:103-110

21. Sharma UC, Pokharel S, van Brakel TJ, van Berlo JH, Cleutjens JP, Schroen B, Andre S, Crijns HJ, Gabius HJ, Maessen J, Pinto YM: Galectin-3 marks activated macrophages in failure-prone hypertrophied hearts and contributes to cardiac dysfunction. Circulation 2004, 110:3121-3128

22. Yu L, Ruifrok WP, Meissner M, Bos EM, van Goor H, Sanjabi B, van der Harst P, Pitt B, Goldstein IJ, Koerts JA, van Veldhuisen DJ, Bank RA, van Gilst WH, Sillje HH, de Boer RA: Genetic and pharmacological inhibition of galectin-3 prevents cardiac remodeling by interfering with myocardial fibrogenesis. Circ Heart Fail 2013, 6: 107-117

23. Calvier L, Martinez-Martinez E, Miana M, Cachofeiro V, Rousseau E, Sadaba JR, Zannad F, Rossignol P, Lopez-Andres N: The impact of galectin-3 inhibition on aldosterone-induced cardiac and renal injuries. JACC Heart Fail 2015, 3:59-67 
24. Committee for the Update of the Guide for the Care and Use of Laboratory Animals; National Research Council: Guide for the Care and Use of Laboratory Animals: Eighth Edition. Washington, DC, National Academies Press, 2011

25. Xia Y, Lee K, Li N, Corbett D, Mendoza L, Frangogiannis NG: Characterization of the inflammatory and fibrotic response in a mouse model of cardiac pressure overload. Histochem Cell Biol 2009, 131: $471-481$

26. Xia Y, Dobaczewski M, Gonzalez-Quesada C, Chen W, Biernacka A, Li N, Lee DW, Frangogiannis NG: Endogenous thrombospondin 1 protects the pressure-overloaded myocardium by modulating fibroblast phenotype and matrix metabolism. Hypertension 2011, 58: 902-911

27. Christia P, Bujak M, Gonzalez-Quesada C, Chen W, Dobaczewski M, Reddy A, Frangogiannis NG: Systematic characterization of myocardial inflammation, repair, and remodeling in a mouse model of reperfused myocardial infarction. J Histochem Cytochem 2013, 61: $555-570$

28. Nagueh SF, Appleton CP, Gillebert TC, Marino PN, Oh JK, Smiseth OA, Waggoner AD, Flachskampf FA, Pellikka PA, Evangelista A: Recommendations for the evaluation of left ventricular diastolic function by echocardiography. J Am Soc Echocardiogr 2009, 22:107-133

29. Saxena A, Bujak M, Frunza O, Dobaczewski M, GonzalezQuesada C, Lu B, Gerard C, Frangogiannis NG: CXCR3-independent actions of the CXC chemokine CXCL10 in the infarcted myocardium and in isolated cardiac fibroblasts are mediated through proteoglycans. Cardiovasc Res 2014, 103:217-227

30. Saxena A, Dobaczewski M, Rai V, Haque Z, Chen W, Li N, Frangogiannis NG: Regulatory $\mathrm{T}$ cells are recruited in the infarcted mouse myocardium and may modulate fibroblast phenotype and function. Am J Physiol Heart Circ Physiol 2014, 307:H1233-H1242

31. Gonzalez-Quesada C, Cavalera M, Biernacka A, Kong P, Lee DW, Saxena A, Frunza O, Dobaczewski M, Shinde A, Frangogiannis NG: Thrombospondin-1 induction in the diabetic myocardium stabilizes the cardiac matrix in addition to promoting vascular rarefaction through angiopoietin-2 upregulation. Circ Res 2013, 113:1331-1344

32. Saxena A, Chen W, Su Y, Rai V, Uche OU, Li N, Frangogiannis NG: IL-1 induces proinflammatory leukocyte infiltration and regulates fibroblast phenotype in the infarcted myocardium. J Immunol 2013, 191:4838-4848

33. Dobaczewski M, Bujak M, Li N, Gonzalez-Quesada C, Mendoza LH, Wang XF, Frangogiannis NG: Smad3 signaling critically regulates fibroblast phenotype and function in healing myocardial infarction. Circ Res 2010, 107:418-428

34. Biernacka A, Cavalera M, Wang J, Russo I, Shinde A, Kong P, Gonzalez-Quesada C, Rai V, Dobaczewski M, Lee DW, Wang XF, Frangogiannis NG: Smad3 signaling promotes fibrosis while preserving cardiac and aortic geometry in obese diabetic mice. Circ Heart Fail 2015, 8:788-798

35. Dobaczewski M, Chen W, Frangogiannis NG: Transforming growth factor (TGF)-beta signaling in cardiac remodeling. J Mol Cell Cardiol 2011, 51:600-606

36. Bujak M, Frangogiannis NG: The role of IL-1 in the pathogenesis of heart disease. Arch Immunol Ther Exp (Warsz) 2009, 57:165-176

37. Cherayil BJ, Weiner SJ, Pillai S: The Mac-2 antigen is a galactosespecific lectin that binds IgE. J Exp Med 1989, 170:1959-1972
38. Kaneko H, Anzai T, Takahashi T, Kohno T, Shimoda M, Sasaki A, Shimizu H, Nagai T, Maekawa Y, Yoshimura K, Aoki H, Yoshikawa T, Okada Y, Yozu R, Ogawa S, Fukuda K: Role of vascular endothelial growth factor-A in development of abdominal aortic aneurysm. Cardiovasc Res 2011, 91:358-367

39. Bujak M, Dobaczewski M, Gonzalez-Quesada C, Xia Y, Leucker T, Zymek P, Veeranna V, Tager AM, Luster AD, Frangogiannis NG: Induction of the $\mathrm{CXC}$ chemokine interferon-gamma-inducible protein 10 regulates the reparative response following myocardial infarction. Circ Res 2009, 105:973-983

40. Rong JX, Shapiro M, Trogan E, Fisher EA: Transdifferentiation of mouse aortic smooth muscle cells to a macrophage-like state after cholesterol loading. Proc Natl Acad Sci U S A 2003, 100: 13531-13536

41. Villarreal FJ, MacKenna DA, Omens JH, Dillmann WH: Myocardial remodeling in hypertensive Ren-2 transgenic rats. Hypertension 1995, 25:98-104

42. Saada A, Reichert F, Rotshenker S: Granulocyte macrophage colony stimulating factor produced in lesioned peripheral nerves induces the up-regulation of cell surface expression of MAC-2 by macrophages and Schwann cells. J Cell Biol 1996, 133:159-167

43. Sakata T, Iwagami S, Tsuruta Y, Teraoka H, Suzuki S, Suzuki R: Role of colony-stimulating factor-1 in macrophage activation in tumor-bearing mice. J Immunol 1992, 149:4002-4008

44. Epelman S, Lavine KJ, Randolph GJ: Origin and functions of tissue macrophages. Immunity 2014, 41:21-35

45. Hubert M, Wang SY, Wang JL, Seve AP, Hubert J: Intranuclear distribution of galectin-3 in mouse 3 T3 fibroblasts: comparative analyses by immunofluorescence and immunoelectron microscopy. Exp Cell Res 1995, 220:397-406

46. Openo KP, Kadrofske MM, Patterson RJ, Wang JL: Galectin-3 expression and subcellular localization in senescent human fibroblasts. Exp Cell Res 2000, 255:278-290

47. Yang RY, Hsu DK, Liu FT: Expression of galectin-3 modulates Tcell growth and apoptosis. Proc Natl Acad Sci U S A 1996, 93: $6737-6742$

48. Akahani S, Nangia-Makker P, Inohara H, Kim HR, Raz A: Galectin3: a novel antiapoptotic molecule with a functional BH1 (NWGR) domain of Bcl-2 family. Cancer Res 1997, 57:5272-5276

49. Li LC, Li J, Gao J: Functions of galectin-3 and its role in fibrotic diseases. J Pharmacol Exp Ther 2014, 351:336-343

50. Hsu DK, Yang RY, Pan Z, Yu L, Salomon DR, Fung-Leung WP, Liu FT: Targeted disruption of the galectin-3 gene results in attenuated peritoneal inflammatory responses. Am J Pathol 2000, 156:1073-1083

51. Mackinnon AC, Gibbons MA, Farnworth SL, Leffler H, Nilsson UJ, Delaine T, Simpson AJ, Forbes SJ, Hirani N, Gauldie J, Sethi T: Regulation of transforming growth factor-beta1-driven lung fibrosis by galectin-3. Am J Respir Crit Care Med 2012, 185:537-546

52. Martinez-Martinez E, Calvier L, Fernandez-Celis A, Rousseau E, Jurado-Lopez R, Rossoni LV, Jaisser F, Zannad F, Rossignol P, Cachofeiro V, Lopez-Andres N: Galectin-3 blockade inhibits cardiac inflammation and fibrosis in experimental hyperaldosteronism and hypertension. Hypertension 2015, 66:767-775

53. de Boer RA, van der Velde AR, Mueller C, van Veldhuisen DJ, Anker SD, Peacock WF, Adams KF, Maisel A: Galectin-3: a modifiable risk factor in heart failure. Cardiovasc Drugs Ther 2014, 28: $237-246$ 